\title{
HIGHER ORDER MEAN CURVATURES OF SAC HALF-LIGHTLIKE SUBMANIFOLDS OF INDEFINITE ALMOST CONTACT MANIFOLDS
}

\author{
FORTUNÉ MASSAMBA AND SAMUEL SSEKAJJA
}

\begin{abstract}
We introduce higher order mean curvatures of screen almost conformal (SAC) half-lightlike submanifolds of indefinite almost contact manifolds, admitting a semi-symmetric non-metric connection. We use them to generalize some known results by Duggal and Sahin on totally umbilical halflightlike submanifolds [Int. J. Math. Math. Sci. 2004, no. 68, 3737-3753]. Also, we derive a new integration formula via the divergence of some special vector fields tangent to these submanifolds, which we later use to characterize minimal and maximal submanifolds. Several examples, where possible, are also included to illustrate the main concepts.
\end{abstract}

\section{INTRODUCTION}

Null (or lightlike) subspaces exist naturally in semi-Riemannian spaces and they play a central role in general relativity. More precisely, in the study of black holes (small volumes of spacetime with infinite density). In fact, they are subspaces whose induced metrics are singular (or simply with vanishing determinants). Differential geometry of these subspaces was introduced by Duggal and Bejancu in their book [6], which was later updated by Dugal and Sahin to [7]. Their approach was later adopted by many other researchers, including but not limited to [3, 8, 9, 12, 13, 14, 17. From the above pieces of work, we can see that the theory of lightlike geometry rests on a number of operators, including shape, Ricci, etc., together with functions constructed from them, like mean curvature, scalar curvature, etc. However, the most important of such functions are the ones derived from algebraic invariants of their respective operators. For instance, trace, determinant, and in a more general sense the $r$-th symmetric functions, $\sigma_{r}$. These functions play a central role in studying higher order mean curvatures in differential geometry of both Riemannian and semi-Riemannian manifolds. In fact, for any given point in a manifold, the $r$-th symmetric function $\sigma_{r}$ coincides with the $r$-th mean

2010 Mathematics Subject Classification. Primary 53D15; Secondary 53C40, 53A10.

Key words and phrases. Half-lightlike submanifold; Screen almost conformal; Newton transformation; Mean curvature.

This work is based on the research supported in part by the National Research Foundation of South Africa (grant numbers 95931 and 106072). 
curvature $S_{r}$. A great deal of work has been done for $r=1$ (see [6, 7, 8, 12] and references therein). But the case $r>1$ is strictly non-linear and complicated. The most efficient way of studying this case is the use of Newton transformations, $T_{r}$, of a given operator $A$ (or a system of operators) which, in some way, linearises $S_{r}$. That is to say, $(-1)^{r-1} r S_{r}=\operatorname{tr}\left(A \circ T_{r-1}\right)$.

Let $M^{m+1}$ be a half-lightlike submanifold of an indefinite contact manifold $\bar{M}^{m+3}$ admitting a semi-symmetric non-metric connection. Then, $M$ carries three shape operators $A_{E}^{*}, A_{N}$ and $A_{W}$, where $E, N$ and $W$ are respectively vector fields in its radical distribution, lightlike transversal bundle, and screen transversal bundle. When the structure vector field $\xi$ is tangent to $M$ but not necessarily in its screen distribution, then $A_{E}^{*}$ is a self-adjoint operator on $T M$ while $A_{N}$ and $A_{W}$ are generally not self-adjoint. If we suppose that $M$ is a screen almost conformal (SAC) [15] half-lightlike submanifold, then the operator $A_{N}$ becomes self-adjoint on $T M$ and therefore diagonalizable on $T M$, and hence we can investigate its higher order mean curvatures. With such mean curvatures, one can also investigate integration geometry on such submanifolds. Integration geometry is fundamentally important as it provides obstructions to the existence of foliations whose leaves enjoy some special geometric properties: totally geodesic (or totally umbilical), minimal, constant mean curvature, and many more. Also, it provides a way of minimizing volume (of submanifolds) as well as energy defined from smooth vector fields on manifolds (see [1] and references therein).

In this paper, we consider a SAC half-lightlike submanifold $M$ of an indefinite contact manifold $\bar{M}$, admitting a semi-symmetric non-metric connection. We derive equations relating the $r$-th mean curvatures and Newton transformations of $A_{N}$ and $A_{E}^{*}$. We generalize some known results for $r=1$ and also derive new integration formulas by computing the divergence of some vector fields on the tangent bundle of $M$. The formula is then used to characterize minimal and $r$-maximal half-lightlike submanifolds. The rest of the paper is arranged as follows. Section 2 outlines the basic preliminary concepts needed in other parts of the paper. Section 3 introduces Newton transformations of $A_{E}^{*}$. In Section 4 we show that the $r$-th mean curvatures and Newton transformations of $A_{N}$ and $A_{E}^{*}$ are in partial variation (see Proposition 4.2 and Theorem 4.4. Also, we derive generalized differential equations for $r$-th mean curvatures (Theorem 4.10). In Section 5 we present special integration formulas by computing the divergence of some vector fields (see Theorem 5.7 and its corollaries).

\section{Preliminaries}

Let $\bar{M}$ be a $(2 n+1)$-dimensional manifold endowed with an almost contact structure $(\bar{\phi}, \xi, \eta)$, i.e. $\bar{\phi}$ is a tensor field of type $(1,1), \xi$ is a vector field, and $\eta$ is a 1-form satisfying

$$
\bar{\phi}^{2}=-\mathbb{I}+\eta \otimes \xi, \quad \eta(\xi)=1, \quad \eta \circ \bar{\phi}=0, \quad \text { and } \quad \bar{\phi}(\xi)=0 .
$$

Then $(\bar{\phi}, \xi, \eta, \bar{g})$ is called an indefinite almost contact metric structure on $\bar{M}$ if $(\bar{\phi}, \xi, \eta)$ is an almost contact structure on $\bar{M}$ and $\bar{g}$ is a semi-Riemannian metric 
on $\bar{M}$ such that (see [4]) for any vector field $\bar{X}, \bar{Y}$ on $\bar{M}$,

$$
\bar{g}(\bar{\phi} \bar{X}, \bar{\phi} \bar{Y})=\bar{g}(\bar{X}, \bar{Y})-\eta(\bar{X}) \eta(\bar{Y}) .
$$

It follows that, for any vector $\bar{X}$ on $\bar{M}, \eta(\bar{X})=\bar{g}(\xi, \bar{X})$. We denote by $\Gamma(\Xi)$ the set of smooth sections of the vector bundle $\Xi$.

A connection $\bar{\nabla}$ on $\bar{M}$ is called a semi-symmetric non-metric connection [11, 17] if $\bar{\nabla}$ and its corresponding torsion tensor $\bar{T}$ satisfy the equations

$$
\left(\bar{\nabla}_{\bar{X}} \bar{g}\right)(\bar{Y}, \bar{Z})=-\eta(\bar{Y}) \bar{g}(\bar{X}, \bar{Z})-\eta(\bar{Z}) \bar{g}(\bar{X}, \bar{Y})
$$

and

$$
\bar{T}(\bar{X}, \bar{Y})=\eta(\bar{Y}) \bar{X}-\eta(\bar{X}) \bar{Y},
$$

for all $\bar{X}, \bar{Y}$ and $\bar{Z}$ vector fields on $\bar{M}$.

Let $(\bar{M}, \bar{g})$ be an $(m+n)$-dimensional semi-Riemannian manifold of constant index $\nu, 1 \leq \nu<m+n$, and let $M$ be a submanifold of $\bar{M}$ of codimension $n$. We assume that both $m$ and $n$ are $\geq 1$. At a point $p \in M$, we define the orthogonal complement $T_{p} M^{\perp}$ of the tangent space $T_{p} M$ by $T_{p} M^{\perp}=\left\{X \in \Gamma\left(T_{p} M\right)\right.$ : $\left.\bar{g}(X, Y)=0, \forall Y \in \Gamma\left(T_{p} M\right)\right\} . \operatorname{Take} \operatorname{Rad} T_{p} M=\operatorname{Rad} T_{p} M^{\perp}=T_{p} M \cap T_{p} M^{\perp}$.

The submanifold $M$ of $\bar{M}$ is said to be an $r$-lightlike submanifold if the mapping $\operatorname{Rad} T M: p \in M \longrightarrow \operatorname{Rad} T_{p} M$ defines a smooth distribution on $M$ of rank $r>0$. We call $\operatorname{Rad} T M$ the radical distribution on $M$.

We say that $M$ is a half-lightlike submanifold of $\bar{M}$ (see [7]) if $r=1, n=2$, and there exist $E, W \in \Gamma\left(T_{p} M^{\perp}\right)$ such that

$$
\bar{g}(E, V)=0, \quad \bar{g}(W, W) \neq 0, \quad \forall V \in \Gamma\left(T_{p} M^{\perp}\right) .
$$

From this, we observe that $E \in \operatorname{Rad} T_{p} M$ and therefore,

$$
\bar{g}(E, X)=\bar{g}(E, V)=0, \quad \forall X \in \Gamma\left(T_{p} M\right), V \in \Gamma\left(T_{p} M^{\perp}\right) .
$$

Thus, $\operatorname{Rad} T M$ is locally (or globally) spanned by $E$.

Let $S(T M)$ be a screen distribution which is a semi-Riemannian complementary distribution of $\operatorname{Rad} T M$ in $T M$, that is,

$$
T M=\operatorname{Rad} T M \perp S(T M) .
$$

Choose a screen transversal bundle $S\left(T M^{\perp}\right)$, which is semi-Riemannian and complementary to $\operatorname{Rad} T M$ in $T M^{\perp}$. Since, for any null section $E$ of $\operatorname{Rad} T M$, there exists a unique null section $N$ of the orthogonal complement of $S\left(T M^{\perp}\right)$ in $S(T M)^{\perp}$ such that $g(E, N)=1$, it follows that there exists a lightlike transversal vector bundle $l \operatorname{tr}(T M)$ locally spanned by $N\left[\underline{6}\right.$. Let $W \in \Gamma\left(S\left(T M^{\perp}\right)\right)$ be a unit vector field, then $\bar{g}(N, N)=\bar{g}(N, Z)=\bar{g}(N, W)=0$, for any $Z \in \Gamma(S(T M))$.

Let $\operatorname{tr}(T M)$ be complementary (but not orthogonal) vector bundle to $T M$ in $T \bar{M}$. Then,

$$
\begin{aligned}
\operatorname{tr}(T M) & =l \operatorname{tr}(T M) \perp S\left(T M^{\perp}\right), \\
T \bar{M} & =S(T M) \perp S\left(T M^{\perp}\right) \perp\{\operatorname{Rad} T M \oplus l \operatorname{tr}(T M)\} .
\end{aligned}
$$


Note that the distribution $S(T M)$ is not unique, and is canonically isomorphic to the factor vector bundle $T M / \operatorname{Rad} T M[6]$.

Let $P$ be the projection of $T M$ onto $S(T M)$. Throughout this paper, we shall suppose that $\xi$ is a unit space-lightlike vector field. Moreover, from $2.3 \xi$ is decomposed as

$$
\xi=\xi_{S}+a E+b N+e W
$$

where $\xi_{S}$ denotes the projection of the tangential part of $\xi$ onto $S(T M)$ and $a=$ $\eta(N), b=\eta(E)$ and $e=\epsilon \eta(W)$, with $\epsilon= \pm 1$, are smooth functions on $\bar{M}$. The Gauss-Weingarten formulas are given by

$$
\begin{gathered}
\bar{\nabla}_{X} Y=\nabla_{X} Y+h(X, Y), \quad \forall X, Y \in \Gamma(T M) \\
\bar{\nabla}_{X} V=-A_{V} X+\nabla_{X}^{t} V, \quad \forall X \in \Gamma(T M), V \in \Gamma(\operatorname{tr}(T M)) .
\end{gathered}
$$

Notice that $\left\{\nabla_{X} Y, A_{V} X\right\}$ and $\left\{h(X, Y), \nabla_{X}^{t} V\right\}$ belong to $\Gamma(T M)$ and $\Gamma(\operatorname{tr}(T M))$ respectively. Further, $\nabla$ and $\nabla^{t}$ are linear connections on $M$ and $\operatorname{tr} T M$, respectively. The second fundamental form $h$ is a symmetric $\mathcal{F}(M)$-bilinear form on $\Gamma(T M)$ with values in $\Gamma(\operatorname{tr}(T M))$ and the shape operator $A_{V}$ is a linear endomorphism of $\Gamma(T M)$. Then, for all $X, Y \in \Gamma(T M)$, 2.5 and 2.6 give

$$
\begin{aligned}
\bar{\nabla}_{X} Y & =\nabla_{X} Y+B(X, Y) N+D(X, Y) W, \\
\bar{\nabla}_{X} N & =-A_{N} X+\tau(X) N+\rho(X) W, \\
\bar{\nabla}_{X} W & =-A_{W} X+\phi(X) N \\
\nabla_{X} P Y & =\nabla_{X}^{*} P Y+C(X, P Y) E, \\
\nabla_{X} E & =-A_{E}^{*} X-\delta(X) E
\end{aligned}
$$

for all $E \in \Gamma(\operatorname{Rad} T M), N \in \Gamma(l \operatorname{tr}(T M))$, and $W \in \Gamma\left(S\left(T M^{\perp}\right)\right)$, where

$$
h(X, Y)=B(X, Y) N+D(X, Y) W
$$

$C$ is the local second fundamental form on $S(T M),\left\{A_{N}, A_{W}\right\}$ and $A_{E}^{*}$ are the shape operators on $T M$ and $S(T M)$ respectively, and $\tau, \rho, \phi$, and $\delta$ are differential 1 -forms on $T M$. Notice that $\nabla^{*}$ is a metric connection on $S(T M)$ while $\nabla$ is generally not a metric connection. In fact, using (2.1) and (2.7), we deduce

$$
\begin{aligned}
\left(\nabla_{X} g\right)(Y, Z)= & B(X, Y) \lambda(Z)+B(X, Z) \lambda(Y) \\
& -\eta(Y) g(X, Z)-\eta(Z) g(X, Y),
\end{aligned}
$$

for all $X, Y, Z \in \Gamma(T M)$, where $\lambda$ is a 1 -form on $T M$ given by $\lambda(\cdot)=\bar{g}(\cdot, N)$. It is well known $([6,7])$ that $B$ and $D$ are independent of the choice of $S(T M)$ and they satisfy

$$
B(X, E)=0, \quad D(X, E)=-\phi(X), \quad \forall X \in \Gamma(T M) .
$$


The three local second fundamental forms $B, D$, and $C$ are related to their shape operators by the following equations:

$$
\begin{gathered}
g\left(A_{E}^{*} X, Y\right)=B(X, Y)-b g(X, Y), \quad \bar{g}\left(A_{E}^{*} X, N\right)=0, \\
g\left(A_{W} X, Y\right)=D(X, Y)-e g(X, Y)+\phi(X) \lambda(Y), \\
g\left(A_{N} X, P Y\right)=C(X, P Y)-a g(X, P Y)-\lambda(X) \eta(P Y), \\
\bar{g}\left(A_{N} X, N\right)=-a \lambda(X), \quad \bar{g}\left(A_{W} X, N\right)=\rho(X)-e \lambda(X), \\
\delta(X)=\tau(X)-b \lambda(X), \quad \forall X, Y \in \Gamma(T M) .
\end{gathered}
$$

From the first equations of 2.12 and 2.13 we deduce that $A_{E}^{*}$ is $S(T M)$-valued, self-adjoint, and satisfies $A_{E}^{*} E=0$. Let $\bar{R}$ and $R$ denote the curvature tensors of $\bar{M}$ and $M$ respectively. Then, using the Gauss-Weingarten equations for $M$, we derive

$$
\begin{aligned}
& \bar{R}(X, Y) Z=R(X, Y) Z+B(X, Z) A_{N} Y-B(Y, Z) A_{N} X+D(X, Z) A_{W} Y \\
& \quad-D(Y, Z) A_{W} X+\left\{\left(\nabla_{X} B\right)(Y, Z)-\left(\nabla_{Y} B\right)(X, Z)+\tau(X) B(Y, Z)\right. \\
& \quad-\tau(Y) B(X, Z)+\phi(X) D(Y, Z)-\phi(Y) D(X, Z)\} N+\left\{\left(\nabla_{X} D\right)(Y, Z)\right. \\
& \left.\quad-\left(\nabla_{Y} D\right)(X, Z)+\rho(X) B(Y, Z)-\rho(Y) B(X, Z)\right\} W \\
& \bar{R}(X, Y) N=-\nabla_{X}\left(A_{N} Y\right)+\nabla_{Y}\left(A_{N} X\right)+A_{N}[X, Y]+\tau(X) A_{N} Y \\
& \quad-\tau(Y) A_{N} X+\rho(X) A_{W} Y-\rho(Y) A_{W} X+\left\{B\left(X, A_{N} X\right)-B\left(X, A_{N} Y\right)\right. \\
& \quad+2 d \tau(X, Y)+\phi(X) \rho(Y)-\phi(Y) \rho(X)\} N+\left\{D\left(X, A_{N} X\right)-D\left(X, A_{N} Y\right)\right. \\
& \quad+2 d \rho(X, Y)+\rho(X) \tau(Y)-\rho(Y) \tau(X)\} W, \quad \forall X, Y \in \Gamma(T M) .
\end{aligned}
$$

A half-lightlike submanifold $M$ of an indefinite almost contact manifold $\bar{M}$, with $\xi \in \Gamma(T M)$, is called screen almost conformal (SAC) [15] if the shape operators $A_{N}$ and $A_{E}^{*}$ of $M$ and $S(T M)$, respectively, are linked to each other by

$$
A_{N}=\varphi A_{E}^{*}+\lambda \otimes \xi
$$

or, equivalently,

$$
C(X, P Y)=\varphi B(X, Y)+\lambda(X) \eta(P Y), \quad \forall X, Y \in \Gamma(T M)
$$

where $\varphi$ is a non-vanishing smooth function on a coordinate neighborhood $\mathcal{U}$ of $M$. Furthermore, $M$ is screen almost homothetic if $\varphi$ is a non-vanishing constant function.

When $\bar{\nabla}$ is a metric connection, it is easy to show that $\bar{g}\left(A_{N} X, N\right)=0$ for any $X \in \Gamma(T M)$, for any half-lightlike submanifold. Hence $A_{N}$ is a screen-valued operator, and thus the screen almost conformality condition 2.18 makes sense only if $\xi \in \Gamma(S(T M))$.

In the following example, we consider the connection in the ambient space to be Levi-Civita and construct a SAC half-lightlike submanifold of an indefinite Kenmotsu manifold. 
In what follows, we consider a manifold $\bar{M}=\left(\mathbb{R}_{q}^{2 m+1}, \bar{\phi}_{0}, \xi, \eta, \bar{g}\right)$ with its usual Kenmotsu structure given by

$$
\begin{aligned}
& \eta=d z, \quad \xi=\partial z, \\
& \bar{g}=\eta \otimes \eta-e^{-2 z} \sum_{i=1}^{\frac{q}{2}}\left(d x^{i} \otimes d x^{i}+d y^{i} \otimes d y^{i}\right)+e^{-2 z} \sum_{i=q+1}^{m}\left(d x^{i} \otimes d x^{i}+d y^{i} \otimes d y^{i}\right), \\
& \bar{\phi}_{0}\left(\sum_{i=1}^{m}\left(X_{i} \partial x^{i}+Y_{i} \partial y^{i}\right)+Z \partial z\right)=\sum_{i=1}^{m}\left(Y_{i} \partial x^{i}-X_{i} \partial y^{i}\right),
\end{aligned}
$$

where $\left(x^{i}, y^{i}, z\right)$ are Cartesian coordinates and $\partial t_{k}=\frac{\partial}{\partial t^{k}}$, for $t \in \mathbb{R}^{2 m+1}$.

Example 2.1. Let $\bar{M}=\left(\mathbb{R}_{2}^{9}, \bar{g}\right)$ be a semi-Euclidean space, where $\bar{g}$ is of signature $(-,+,+,+,-,+,+,+,+)$ with respect to the canonical basis

$$
\left(\partial x_{1}, \partial x_{2}, \partial x_{3}, \partial x_{4}, \partial y_{1}, \partial y_{2}, \partial y_{3}, \partial y_{4}, \partial z\right) \text {. }
$$

Let $\bar{\nabla}$ be the Levi-Civita connection with respect to the semi-Riemannian metric $\bar{g}$ and consider the vector fields $u_{1}, \ldots, u_{9}$, where for all $1 \leq i \leq 8$ we have

$$
\begin{aligned}
u_{i}= & e^{z} \sum_{\alpha^{\prime}=1}^{4} f_{i \alpha^{\prime}}\left(x_{1}, \ldots, x_{4}, y_{1}, \ldots, y_{4}, z\right) \partial x_{\alpha^{\prime}} \\
& +e^{z} \sum_{\beta^{\prime}=\alpha^{\prime}+1}^{8} f_{i \beta^{\prime}}\left(x_{1}, \ldots, x_{4}, y_{1}, \ldots, y_{4}, z\right) \partial y_{\beta^{\prime}}, \operatorname{det}\left(f_{i j}\right) \neq 0, \\
u_{9}= & -\xi
\end{aligned}
$$

where functions $f_{i \alpha^{\prime}}$ and $f_{i \beta^{\prime}}$ are defined such that the action of the connection $\bar{\nabla}$ on the basis $\left\{u_{1}, \ldots, u_{9}\right\}$ gives $\bar{\nabla}_{u_{i}} u_{i}=\xi$ for all $i=1,5, \bar{\nabla}_{u_{j}} u_{j}=-\xi$ for all $j=2,3,4,6,7,8, \bar{\nabla}_{u_{3}} u_{1}=e^{z} u_{5}, \bar{\nabla}_{u_{3}} u_{5}=-e^{z} u_{1}, \bar{\nabla}_{u_{7}} u_{1}=e^{z} u_{5}, \bar{\nabla}_{u_{7}} u_{5}=-e^{z} u_{1}$, $\bar{\nabla}_{u_{1}} u_{3}=e^{z} u_{5}, \bar{\nabla}_{u_{1}} u_{7}=e^{z} u_{5}, \bar{\nabla}_{u_{5}} u_{3}=-e^{z} u_{1}, \bar{\nabla}_{u_{5}} u_{7}=e^{z} u_{1}$, and the rest of the connections $\bar{\nabla}_{u_{i}} u_{j}=0$ for all $i \neq j$, where $i, j=1, \ldots, 8$. Furthermore, the nonvanishing brackets are $\left[u_{i}, u_{9}\right]=u_{i}$, for all $i=1, \ldots, 8$. Using Koszul's formula, we have $\bar{\nabla}_{u_{i}} u_{9}=u_{i}$ for all $i=1, \ldots, 8$ and $\bar{\nabla}_{u_{9}} u_{9}=0$. From these constructions, $\left(\bar{\phi}_{0}, u_{9}, \eta, \bar{g}\right)$ defines an almost contact structure on $\mathbb{R}_{2}^{9}$. Therefore, $\left(\mathbb{R}_{2}^{9}, \bar{\phi}_{0}, u_{9}, \eta, \bar{g}\right)$ is an indefinite Kenmotsu manifold.

Next, let us consider a submanifold $M$ of $\mathbb{R}_{2}^{9}$ above which is given by the equation $x_{1}=\sqrt{2}\left(x_{2}+y_{2}\right)$. By straightforward calculations, one can easily show that the vectors $E=\frac{1}{\sqrt{2}}\left(u_{6}+u_{2}\right)-u_{1}, Z_{1}=u_{3}, Z_{2}=u_{7}, Z_{3}=u_{6}-u_{2}, Z_{4}=u_{5}, Z_{5}=u_{8}$, and $Z_{6}=u_{9}=-\xi$ form a local frame of $T M$. Clearly, $\operatorname{Rad} T M=\operatorname{span}\{E\}$ and $S(T M)=\operatorname{span}\left\{Z_{1}, \ldots, Z_{6}\right\}$. Also, the lightlike transversal bundle $l \operatorname{tr}(T M)$ and co-screen $S\left(T M^{\perp}\right)$ are respectively spanned by $N$ and $W$, where $N=\frac{1}{2 \sqrt{2}}\left(u_{6}+\right.$ $\left.u_{2}\right)+\frac{1}{2} u_{1}$ and $W=U_{4}$. Thus, $M$ is a half-lightlike submanifold of $\left(\mathbb{R}_{2}^{9}, \bar{\phi}_{0}, u_{9}, \eta, \bar{g}\right)$. By straightforward calculations, we have $\bar{\nabla}_{Z_{1}} N=\frac{1}{2} e^{z} u_{5}, \bar{\nabla}_{Z_{2}} N=\frac{1}{2} e^{z} u_{5}, \bar{\nabla}_{Z_{3}} N=$ $\bar{\nabla}_{Z_{4}} N=\bar{\nabla}_{Z_{5}} N=\bar{\nabla}_{Z_{6}} N=0, \bar{\nabla}_{E} N=-u_{9}=\xi$. Furthermore, $\nabla_{Z_{1}} E=-\frac{1}{2} e^{z} u_{5}$, $\nabla_{Z_{2}} E=-\frac{1}{2} e^{z} u_{5}, \nabla_{Z_{3}} E=\nabla_{Z_{4}} E=\nabla_{Z_{5}} E=\nabla_{Z_{6}} E=\nabla_{E} E=0$. From these 
connections, the 1-forms $\tau$ and $\rho$ vanish on $T M$. Therefore, from (2.8) and 2.10) we deduce that $A_{N} Z_{1}=-\frac{1}{2} e^{z} u_{5}, A_{N} Z_{2}=-\frac{1}{2} e^{z} u_{5}, A_{N} Z_{3}=A_{N} Z_{4}=A_{N} Z_{5}=$ $A_{N} Z_{6}=0, A_{N} E=u_{9}=-\xi$. Also, $A_{E}^{*} Z_{1}=e^{z} u_{5}, A_{E}^{*} Z_{2}=e^{z} u_{5}, A_{E}^{*} Z_{3}=$ $A_{E}^{*} Z_{4}=A_{E}^{*} Z_{5}=A_{E}^{*} Z_{6}=A_{E}^{*} E=0$. From these relations, we deduce that $A_{N} X=\varphi A_{E}^{*} X+\lambda(X) \xi$ for all $X \in \Gamma(T M)$, with $\varphi=-\frac{1}{2}$. Hence, $M$ is SAC half-lightlike submanifold of $\left(\mathbb{R}_{2}^{9}, \bar{\phi}_{0}, u_{9}, \eta, \bar{g}\right)$.

When $\bar{\nabla}$ is a semi-symmetric non-metric connection, one can easily verify that $\bar{g}\left(A_{N} X, N\right)=-\eta(N) \lambda(X)$, for any $X \in \Gamma(T M)$. See 2.15 for details. This shows that $A_{N}$ is generally not a screen-valued operator. Thus, the screen almost conformality condition (2.18) allows a $\xi \in \Gamma(T M)$ but not necessarily in $S(T M)$, given by $\xi=\xi_{S}+a E$. Since on any SAC half-lightlike submanifold we have $\xi \in \Gamma(T M)$, it is easy to see from (2.4) that $b=0$ and $e=0$. Hence, using (2.14) and 2.19 we deduce that $M$ is a SAC half-lightlike submanifold of $\bar{M}$ admitting a semi-symmetric non-metric connection if and only if

$$
A_{N} X=\varphi A_{E}^{*} X-a X, \quad \forall X \in \Gamma(T M) .
$$

Furthermore, if $M$ is a SAC half-lightlike submanifold of an almost contact manifold $\bar{M}$ admitting a semi-symmetric non-metric connection, then from $(2.20)$ the operator $A_{N}$ is self-adjoint on $T M$ and thus diagonalizable on $T M$. Also, from 2.20 we have that any SAC half-lightlike submanifold of an almost contact manifold admitting a semi-symmetric non-metric connection, with the structure vector field $\xi \in \Gamma(S(T M))$, is screen conformal.

\section{Newton transformations of $A_{E}^{*}$}

Let $\bar{M}$ be an $(n+3)$-dimensional almost contact metric manifold admitting a semi-symmetric non-metric connection and $(M, g, S(T M))$ be a codimension two SAC half-lightlike submanifold of $\bar{M}$. Since $A_{E}^{*}$ is a self-adjoint operator, from (2.13) and 2.20 we can see that $A_{N}$ is a self-adjoint linear operator on $T M$. Thus, $A_{E}^{*}$ and $A_{N}$ are diagonalizable. Hence, $A_{E}^{*}$ has $n+1$ real eigenvalues $\kappa_{0}^{*}=$ $0, \kappa_{1}^{*}, \ldots, \kappa_{n}^{*}$ (the principal curvatures) corresponding to a set of quasi-orthonormal frame field of eigenvector fields $\left\{Z_{0}=E, Z_{1}, \ldots, Z_{n}\right\}$. By the SAC condition 2.20) it is easy to see that $-a,\left(\varphi \kappa_{1}^{*}-a\right), \ldots,\left(\varphi \kappa_{n}^{*}-a\right)$ are eigenvalues of $A_{N}$. Moreover the matrix of $A_{N}$ has the form

$$
A_{N}=\operatorname{diag}\left(-a, \varphi \kappa_{1}^{*}-a, \ldots, \varphi \kappa_{n}^{*}-a\right) .
$$

Associated to the shape operator $A_{E}^{*}$ are $n+1$ algebraic invariants

$$
S_{r}^{*}=\sigma_{r}\left(\kappa_{0}^{*}, \kappa_{1}^{*}, \ldots, \kappa_{n}^{*}\right),
$$

where $\sigma_{r}: M^{n+1} \rightarrow \mathbb{R}$, for $r=0,1, \ldots, n+1$, are symmetric functions given by

$$
\sigma_{r}\left(\kappa_{0}^{*}, \kappa_{1}^{*}, \ldots, \kappa_{n}^{*}\right)=\sum_{0 \leq i_{1}<\cdots<i_{r} \leq n} \kappa_{i_{1}}^{*} \cdots \kappa_{i_{r}}^{*} .
$$


Let us denote by $\mathbb{I}$ the identity map in $\Gamma(T M)$. Then, the characteristic polynomial of $A_{E}^{*}$ is given by

$$
Q(t)=\operatorname{det}\left(A_{E}^{*}-t \mathbb{I}\right)=\sum_{\alpha=0}^{n+1}(-1)^{\alpha} S_{r}^{*} t^{n+1-\alpha} .
$$

The normalized $r$-th mean curvature $H_{r}^{*}$ of $M$ is defined by

$$
\left.H_{r}^{*}=\left(\begin{array}{c}
n+1 \\
r
\end{array}\right)^{-1} S_{r}^{*} \text { and } H_{0}^{*}=1 \text { (a constant function } 1\right) .
$$

In particular, when $r=1$, then $H_{1}^{*}=\frac{1}{n+1} \operatorname{tr}\left(A_{E}^{*}\right)$, which is called the mean curvature of the half-lightlike submanifold $M$. On the other hand, $H_{2}^{*}$ relates directly with the (intrinsic) scalar curvature of $M$. Often times, $H_{r}^{*}$, instead of $S_{r}^{*}$, is called the $r$-th mean curvature [3, 2]. Moreover, the functions $S_{r}^{*}\left(H_{r}^{*}\right)$ are smooth on the whole $M$ and, for any point $p \in M, S_{r}^{*}$ coincides with the $r$-th mean curvature at $p$. Throughout this paper, we shall use $S_{r}^{*}$ instead of $H_{r}^{*}$.

The Newton transformations $T_{r}^{*}: \Gamma(T M) \rightarrow \Gamma(T M)$, for $r=0,1, \ldots, n+1$, of a SAC half-lightlike submanifold $M$ of an $(n+3)$-dimensional almost contact metric manifold $\bar{M}$ with respect to $A_{N}$ are given by

$$
T_{r}^{*}=\sum_{\alpha=0}^{r}(-1)^{\alpha} S_{\alpha}^{*} A_{E}^{* r-\alpha},
$$

or equivalently by the inductive formula

$$
T_{0}^{*}=\mathbb{I}, \quad T_{r}^{*}=(-1)^{r} S_{r}^{*} \mathbb{I}+A_{E}^{*} \circ T_{r-1}^{*}, \quad 1 \leq r \leq n .
$$

Notice that, by the Cayley-Hamiliton theorem, we have $T_{n+1}^{*}=0$. Moreover, $T_{r}^{*}$ are also self-adjoint and commute with $A_{E}^{*}$.

It is important to note that the operators $T_{r}^{*}$ depend on the choice of the transversal bundle $\operatorname{tr}(T M)$ and the screen distribution $S(T M)$. Suppose a screen distribution $S(T M)$ changes to another screen $S(T M)^{\prime}$. The following are some of the local transformation equations due to this change (see [6, p. 87] for more details):

$$
\begin{aligned}
W_{i}^{\prime} & =\sum_{j=1}^{n} W_{i}^{j}\left(W_{j}-\epsilon_{j} c_{j} E\right), \\
N^{\prime}(X) & =N-\frac{1}{2} g(W, W) E+W, \\
A_{E}^{\prime} X & =A_{E}^{*} X+B\left(X, N-N^{\prime}\right) E, \\
\nabla_{X}^{\prime} Y & =\nabla_{X} Y+B(X, Y)\left\{\frac{1}{2} g(W, W) E-W\right\},
\end{aligned}
$$

for any $X, Y \in \Gamma\left(\left.T M\right|_{\mathcal{U}}\right)$, where $W=\sum_{i=1}^{n} c_{i} W_{i},\left\{W_{i}\right\}$ and $\left\{W_{i}^{\prime}\right\}$ are the local orthonormal bases of $S(T M)$ and $S(T M)^{\prime}$ with respective transversal sections $N$ and $N^{\prime}$ for the same null section $B$. Here $c_{i}$ and $W_{i}^{j}$ are smooth functions on $\mathcal{U}$ and $\left\{\epsilon_{1}, \ldots, \epsilon_{n}\right\}$ is the signature of the basis $\left\{W_{1}, \ldots, W_{n}\right\}$. Denote by $\omega$ the dual 
1-form of $W$, characteristic vector field of the screen change, with respect to the induced metric $g=\left.\bar{g}\right|_{M}$, that is,

$$
\omega(X)=g(X, W), \quad \forall X \in \Gamma(T M) .
$$

Consider an orthogonal basis $\left\{Z_{i}\right\}$, for $i \in\{1, \ldots, n\}$, which diagonalizes $A_{E}^{\prime *}$ and $A_{E}^{*}$. Let $k_{i}^{\prime}$ and $k_{i}$ be the eigenvalues corresponding to the eigenvector $Z_{i}$. Then, from (3.6) we have $\left(k_{i}^{\prime}-k_{i}\right) Z_{i}=-B\left(Z_{i}, W\right) E$, which shows that the eigenvalues change under the change of the screen distribution. Since the generalized expansion $\Theta_{r}$ depends on the eigenvalues $k_{i}$, i.e. $\Theta_{r}=(-1)^{r} S_{r}^{*}=(-1)^{r} \sigma_{r}\left(k_{1}, \ldots, k_{n}\right)$, then a change of $N$ will cause a change in it. Now, let $\left\{\Theta, T_{r}^{*}\right\}$ and $\left\{\Theta^{\prime}, T_{r}^{*^{\prime}}\right\}$ be two sets of the above objects under a change in $N$. Applying the recurrence relation (3.3) and the fact that $T_{r} Z_{i}=(-1)^{r} S_{r}^{* i} Z_{i}$, we have

$$
\begin{aligned}
T_{r}^{*^{\prime}} Z_{i} & =\Theta_{r}^{\prime} \mathbb{I}+(-1)^{r-1} S_{r-1}^{* i^{\prime}} A_{E}^{*^{\prime}} Z_{i}, \\
T_{r}^{*} Z_{i} & =\Theta_{r} \mathbb{I}+(-1)^{r-1} S_{r-1}^{* i} A_{E}^{*} Z_{i} .
\end{aligned}
$$

Subtracting the second relation in $(3.9)$ from the first and using relation $(3.6)$ with $X=Z_{i}$, we deduce that the operators $T_{r}^{*}$ and $T_{r}^{*^{\prime}}$ are related by the equation

$$
T_{r}^{*^{\prime}}=T_{r}+\left(\Theta_{r}^{\prime}-\Theta_{r}\right) \mathbb{I}+\theta_{r} A_{E}^{*}+B\left(T_{r-1}^{*^{\prime}}, N-N^{\prime}\right) E
$$

where $\theta_{r}:=(-1)^{r-1}\left(S_{r-1}^{* i^{\prime}}-S_{r-1}^{* i}\right)$. It is easy to see that the tensor $T_{r}^{*}$ is unique if and only if the null hypersurface $M$ is totally geodesic. For more details on Newton transformations and their properties, we refer the reader to [2, [1] and many more references therein.

Let $S_{r}^{* \beta}=\sigma_{r}\left(\kappa_{0}^{*}, \kappa_{1}^{*}, \ldots, \kappa_{\beta-1}^{*}, \kappa_{\beta+1}^{*}, \ldots, \kappa_{n}^{*}\right)$ for $1 \leq \beta \leq n$. A half-lightlike submanifold $M$ of an $(n+3)$-dimensional almost contact metric manifold $\bar{M}$ is called $r$-umbilical (resp. $r$-maximal) [3] if for all $i, j \in\{1, \ldots, n\}$, we have

$$
S_{r}^{* i}=S_{r}^{* j} \quad\left(\text { resp. } H_{r}^{*}=0\right), \quad 1 \leq r \leq n .
$$

Then, the following algebraic properties of $T_{r}^{*}$ are well-known (see [3, 2, 1] and references therein for details):

$$
\begin{aligned}
T_{r}^{*} Z_{\beta} & =(-1)^{r} S_{r}^{* \beta} Z_{\beta}, \\
\operatorname{tr}\left(T_{r}^{*}\right) & =(-1)^{r}(n+1-r) S_{r}^{*}, \\
\operatorname{tr}\left(A_{E}^{*} \circ T_{r-1}^{*}\right) & =(-1)^{r-1} r S_{r}^{*}, \\
\operatorname{tr}\left(A_{E}^{* 2} \circ T_{r-1}^{*}\right) & =(-1)^{r}\left(-S_{1}^{*} S_{r}^{*}+(r+1) S_{r+1}^{*}\right), \\
\operatorname{tr}\left(T_{r-1}^{*} \circ \nabla_{X} A_{E}^{*}\right) & =(-1)^{r-1} X\left(S_{r}^{*}\right), \quad \forall X \in \Gamma(T M) .
\end{aligned}
$$

Next, we define the divergence of the operator $T_{r}^{*}: \Gamma(T M) \rightarrow \Gamma(T M)$ as the vector field $\operatorname{div}^{\nabla}\left(T_{r}^{*}\right) \in \Gamma(T M)$ and given by

$$
\operatorname{div}^{\nabla}\left(T_{r}^{*}\right)=\operatorname{tr}\left(\nabla T_{r}^{*}\right)=\sum_{\beta=0}^{n}\left(\nabla_{Z_{\beta}} T_{r}^{*}\right) Z_{\beta} .
$$

In line with 3.12 , we can see that the SAC half-lightlike submanifold given in Example 2.1 is $r$-minimal with $1 \leq r \leq 6$. 


\section{Fundamental SaC equations of $A_{N}$}

In this section, we derive SAC equations of $A_{N}$ from those of $A_{E}^{*}$. We use some of them to generalize some known results of [8]. Let $T M=\operatorname{span}\left\{Z_{0}=E, Z_{1}, \ldots, Z_{n}\right\}$ and $S(T M)=\operatorname{span}\left\{Z_{1}, \ldots, Z_{n}\right\}$.

Proposition 4.1. Let $M$ be a $S A C$ half-lightlike submanifold of an almost contact manifold $\bar{M}$ admitting a semi-symmetric non-metric connection. Let $S_{1}$ and $S_{1}^{*}$ be the first order mean curvatures corresponding to the two shape operators $A_{N}$ and $A_{E}^{*}$ respectively. Then,

$$
S_{1}=\varphi S_{1}^{*}-a n
$$

Proof. From $H_{1}=\frac{1}{n+1} \operatorname{tr}\left(A_{N}\right)$ and $H_{1}=\left(\begin{array}{c}n+1 \\ 1\end{array}\right)^{-1} S_{1}$, we have $S_{1}=\operatorname{tr}\left(A_{N}\right)$. From the last equation and the fact that $M$ is a SAC half-lightlike submanifold, we have $S_{1}=\left.\operatorname{tr}\left(A_{N}\right)\right|_{S(T M)}=\varphi \operatorname{tr}\left(A_{E}^{*}\right)-$ an, which completes the proof.

In the next proposition, we generalize Proposition 4.1

Proposition 4.2. Let $M$ be a SAC half-lightlike submanifold of an almost contact manifold $\bar{M}$ admitting a semi-symmetric non-metric connection. Let $S_{r}$ and $S_{r}^{*}$ be the r-th mean curvatures corresponding to the two shape operators $A_{N}$ and $A_{E}^{*}$ respectively. Then, for all $r \geq 1$ we have

$$
S_{r}=\varphi^{r} S_{r}^{*}+J_{r}^{*}(a, \varphi),
$$

where for a given $A_{E}^{*}, J_{r}^{*}$ are smooth functions in a and $\varphi$ given by

$$
\begin{aligned}
J_{r}^{*}(a, \varphi)= & \sum_{0<i_{1}<\cdots<i_{r} \leq n}(-1)^{r} a^{r} \\
& +\sum_{0 \leq i_{1}<\cdots<i_{r} \leq n} \sum_{j=1}^{r-1}(-1)^{r+j} e_{j}\left(\kappa_{i 1}^{*}, \ldots, \kappa_{i r}^{*}\right) a^{r-j} \varphi^{j} .
\end{aligned}
$$

Proof. Let $\kappa_{0}^{*}, \ldots, \kappa_{n}^{*}$ be the eigenvalues (principal curvatures) of $A_{E}^{*}$ and consider a linear factorization of a $k^{\text {th }}$-degree monic polynomial in $t$ below:

$$
\prod_{i=1}^{k}\left(t-X_{i}\right)=\sum_{s=0}^{k}(-1)^{s} e_{s}\left(X_{1}, \ldots, X_{k}\right) t^{k-s}
$$

where $e_{s}$ denotes the $s^{\text {th }}$-degree symmetric function in the variables $X_{1}, \ldots, X_{k}$. Thus, if $S_{r}$ is the $r$-th mean curvature of $A_{N}$, then we have from the definition of 
$S_{r}$ and (4.4) that

$$
\begin{aligned}
S_{r}= & \sum_{0 \leq i_{1}<\cdots<i_{r} \leq n} \kappa_{i_{1}} \cdots \kappa_{i_{r}} \\
= & \sum_{0 \leq i_{1}<\cdots<i_{r} \leq n} \prod_{j=1}^{r}\left(\varphi \kappa_{i_{j}}^{*}-a\right) \\
= & \varphi^{r} \sum_{0 \leq i_{1}<\cdots<i_{r} \leq n} e_{r}\left(\kappa_{i_{1}}^{*}, \ldots, \kappa_{i_{r}}^{*}\right)+\sum_{0<i_{1}<\cdots<i_{r} \leq n}(-1)^{r} a^{r} \\
& +\sum_{0 \leq i_{1}<\cdots<i_{r} \leq n}(-1)^{r}\left\{-e_{1}\left(\kappa_{i_{1}}^{*}, \ldots, \kappa_{i r}^{*}\right) a^{r-1} \varphi+\cdots\right. \\
& \left.+(-1)^{r-1} e_{r-1}\left(\kappa_{i_{1}}^{*}, \ldots, \kappa_{i_{r}}^{*}\right) a \varphi^{r-1}\right\} \\
= & \varphi^{r} S_{r}^{*}+\sum_{0<i_{1}<\cdots<i_{r} \leq n}(-1)^{r} a^{r} \\
& +\sum_{0 \leq i_{1}<\cdots<i_{r} \leq n} \sum_{j=1}^{r-1}(-1)^{r+j} e_{j}\left(\kappa_{i_{1}}^{*}, \ldots, \kappa_{i_{r}}^{*}\right) a^{r-j} \varphi^{j},
\end{aligned}
$$

which proves 4.2 and 4.3 , hence the proposition.

Notice that $J_{r}^{*}(0, \varphi)=0$, which is the case when the structure vector field $\xi$ belongs to $S(T M)$. From 4.2 , we have $S_{1}=\varphi S_{1}^{*}+J_{1}^{*}(a, \varphi)$ and from (4.3), we can see that $J_{1}^{*}(a, \varphi)=-a n$. Thus, $S_{1}=\varphi S_{1}^{*}-a n$, which is Proposition 4.1

In what follows, we construct a SAC half-lightlike submanifold in which $\xi \in$ $\Gamma(S(T M))$ of an indefinite almost contact manifold $\bar{M}=\mathbb{R}_{2}^{9}$, admitting a semisymmetric non-metric connection. Notice from 2.20 that, under the above conditions, we have $a=0$ and therefore the corresponding SAC half-lightlike submanifold is actually screen conformal.

Example 4.3. Let $\bar{M}=\left(\mathbb{R}_{2}^{9}, \bar{\phi}_{0}, \xi, \eta, \bar{g}\right)$ be an almost contact manifold, where $\bar{g}$ is of signature $(-,-,+,+,+,+,+,+,+)$ with respect to the canonical basis

$$
\left(\partial x_{1}, \partial x_{2}, \partial x_{3}, \partial x_{4}, \partial x_{5}, \partial x_{6}, \partial x_{7}, \partial x_{8}, \partial z\right)
$$

where $\left(x_{1}, \ldots, x_{8}, z\right)$ are the usual coordinates on $\bar{M}$. Let $\bar{\phi}_{0} \partial x_{1}=\partial x_{2}, \bar{\phi}_{0} \partial x_{2}=$ $-\partial x_{1}, \bar{\phi}_{0} \partial x_{3}=\partial x_{4}, \bar{\phi}_{0} \partial x_{3}=-\partial x_{4}, \bar{\phi}_{0} \partial x_{5}=\partial x_{6}, \bar{\phi}_{0} \partial x_{6}=-\partial x_{5}, \bar{\phi}_{0} \partial x_{7}=\partial x_{8}$, $\bar{\phi}_{0} \partial x_{8}=-\partial x_{7}$, and $\bar{\phi}_{0} \partial z=0$. Consider a submanifold of $\bar{M}$ defined by

$$
M=\left\{\left(x_{1}, \ldots, x_{8}, z\right) \in \mathbb{R}_{2}^{9}: x_{1}=x_{2}-x_{7}-x_{8}\right\} .
$$

Following simple calculations, we can see that the vector $E=\partial x_{1}-\partial x_{2}+\partial x_{7}+\partial x_{8}$ is lightlike with corresponding lightlike transversal vector $N=-\frac{1}{4}\left(\partial x_{1}-\partial x_{2}-\partial x_{7}-\right.$ $\left.\partial x_{8}\right)$. Hence, $\operatorname{Rad} T M=\operatorname{span}\{E\}$ and $l \operatorname{tr}(T M)=\operatorname{span}\{N\}$. Furthermore, the vector fields $Z_{1}=e^{x_{1}} \partial x_{3}, Z_{2}=e^{x_{1}} \partial x_{4}, Z_{3}=e^{x_{1}} \partial x_{6}, Z_{4}=\partial x_{1}+\partial x_{2}-\partial x_{7}+\partial x_{8}$, $Z_{5}=-\frac{1}{4}\left(\partial x_{1}+\partial x_{2}+\partial x_{7}-\partial x_{8}\right)$, and $Z_{6}=\partial z=\xi$, span $S(T M)$. Also, $S(T M=$ $\operatorname{span}\{W\}$, where $W=-e^{x_{1}} \partial x_{5}$. Hence, $M$ is a half-lightlike submanifold of $\bar{M}$. By straightforward calculations, we have $[E, N]=0,\left[Z_{j}, N\right]=0$, for $j=4,5,6$ and $\left[Z_{i}, N\right]=\frac{1}{4} Z_{i}$, for $i=1,2,3$. In a similar way, we have $\left[Z_{j}, E\right]=0$ for $j=4,5,6$ 
and $\left[Z_{i}, E\right]=-Z_{i}$, for $i=1,2,3$. Further, $\left[Z_{1}, Z_{4}\right]=-Z_{1},\left[Z_{1}, Z_{5}\right]=\frac{1}{4} Z_{1}$, $\left[Z_{2}, Z_{4}\right]=-Z_{2},\left[Z_{2}, Z_{5}\right]=\frac{1}{4} Z_{2},\left[Z_{3}, Z_{4}\right]=-Z_{3},\left[Z_{3}, Z_{5}\right]=\frac{1}{4} Z_{3}$. Additionally, $[W, E]=-W$ and $[W, N]=\frac{1}{4} W$. All other brackets vanish. Notice that $S(T M)$ is integrable. Using the fact that $\bar{\nabla}$ is a semi-symmetric non-metric connection, we get $\tau=\rho=0, \bar{\nabla}_{E} N=0, \bar{\nabla}_{Z_{1}} N=\frac{1}{4} Z_{1}, \bar{\nabla}_{Z_{2}} N=\frac{1}{4} Z_{2}, \bar{\nabla}_{Z_{3}} N=\frac{1}{4} Z_{3}$, $\bar{\nabla}_{Z_{j}} N=0$, for $j=4,5,6$. Also, $\phi=0, \nabla_{E} E=0, \nabla_{Z_{1}} E=-Z_{1}, \nabla_{Z_{2}} E=-Z_{2}$, $\nabla_{Z_{3}} E=-Z_{3}, \nabla_{Z_{j}} E=0$, for $j=4,5,6$. From these calculations, we deduce that $A_{N} X=-\frac{1}{4} A_{E}^{*} X$, for all $X \in \Gamma(T M)$. Thus $M$ is a SAC (in particular, screen homothetic) half-lightlike submanifold, with $\varphi=-\frac{1}{4}$ and $a=0$, of $\bar{M}$ admitting a semi-symmetric non-metric connection. Further still, if $k_{0}^{*}, \ldots, k_{6}^{*}$ and $k_{0}, \ldots, k_{6}$ are the principal curvatures of $A_{E}^{*}$ and $A_{N}$ with respect to the basis of eigenvectors $\left\{E, Z_{1}, \ldots, Z_{6}\right\}$, respectively, then from the above information we have $k_{0}^{*}=0, k_{1}^{*}=1, k_{2}^{*}=1, k_{3}^{*}=1, k_{4}^{*}=0, k_{5}^{*}=0, k_{6}^{*}=0$ and $k_{0}=0$, $k_{1}=-\frac{1}{4}, k_{2}=-\frac{1}{4}, k_{3}=-\frac{1}{4}, k_{4}=0, k_{5}=0, k_{6}=0$. Hence, the matrices of $A_{E}^{*}$ and $A_{N}$ are respectively the diagonal matrices given by $\operatorname{diag}(0,1,1,1,0,0,0)$ and $\operatorname{diag}\left(0,-\frac{1}{4},-\frac{1}{4},-\frac{1}{4}, 0,0,0\right)$. Furthermore,

$$
S_{r}^{*}=\sigma_{r}\left(k_{0}^{*}, \ldots, k_{6}^{*}\right)=\sigma_{r}(0,1,1,1,0,0,0), \quad 1 \leq r \leq 6 .
$$

Notice that $S_{0}^{*}=1, S_{1}^{*}=3, S_{2}^{*}=3$, etc. In a similar way, if $S_{r}$ is the $r$-th mean curvature with respect to $A_{N}$, then

$$
S_{r}=\sigma_{r}\left(k_{0}, \ldots, k_{6}\right)=\sigma_{r}\left(0,-\frac{1}{4},-\frac{1}{4},-\frac{1}{4}, 0,0,0\right)=\left(-\frac{1}{4}\right)^{r} S_{r}^{*}, \quad 1 \leq r \leq 6 .
$$

Finally, we notice that the mean curvatures $S_{r}^{*}$ and $S_{r}$ are also conformally related, i.e., $S_{r}=\varphi^{r} S_{r}^{*}$ and $J_{r}^{*}\left(0,-\frac{1}{4}\right)=0$.

Theorem 4.4. Let $M$ be a SAC half-lightlike submanifold of an almost contact manifold $\bar{M}$ admitting a semi-symmetric non-metric connection. Let $T_{r}$ and $T_{r}^{*}$ be the $r$-th Newton transformations corresponding to the two shape operators $A_{N}$ and $A_{E}^{*}$ respectively. Then, for all $r \geq 1$ we have

$$
T_{r}=\varphi^{r} T_{r}^{*}+\mathcal{N}_{r}^{*}(a, \varphi)
$$

where $\mathcal{N}_{r}^{*}$ are operators depending on $a, \varphi$, and $A_{E}^{*}$ given by

$$
\begin{aligned}
\mathcal{N}_{r}^{*}(a, \varphi)= & \sum_{\alpha=1}^{r}(-1)^{\alpha}\left\{J_{\alpha}^{*}(a, \varphi)\left(\varphi A_{E}^{*}-a \mathbb{I}\right)^{r-\alpha}\right. \\
& \left.+\varphi^{\alpha} S_{\alpha}^{*} \sum_{k=1}^{r-\alpha}(-1)^{k}\left(\begin{array}{c}
r-\alpha \\
k
\end{array}\right)\left(\varphi A_{E}^{*}\right)^{r-\alpha-k}(a \mathbb{I})^{k}\right\} \\
& +\sum_{j=1}^{r}(-1)^{j}\left(\begin{array}{c}
r \\
j
\end{array}\right)\left(\varphi A_{E}^{*}\right)^{r-j}(a \mathbb{I})^{j} .
\end{aligned}
$$


Proof. Using the fact that $M$ is SAC half-lightlike submanifold, the definition of $T_{r}, 3.2$, and Proposition 4.2 we get

$$
\begin{aligned}
T_{r} & =\sum_{\alpha=0}^{r}(-1)^{\alpha} S_{\alpha} A_{N}^{r-\alpha}=A_{N}^{r}+\sum_{\alpha=1}^{r}(-1)^{\alpha} S_{\alpha} A_{N}^{r-\alpha} \\
& =\left(\varphi A_{E}^{*}-a \mathbb{I}\right)^{r}+\sum_{\alpha=1}^{r}(-1)^{\alpha}\left(\varphi^{\alpha} S_{\alpha}^{*}+J_{\alpha}^{*}(a, \varphi)\right)\left(\varphi A_{E}^{*}-a \mathbb{I}\right)^{r-\alpha} .
\end{aligned}
$$

Applying the binomial theorem, the above equation leads to

$$
\begin{aligned}
T_{r} & =\varphi^{r} A_{E}^{* r}+\sum_{j=1}^{r}(-1)^{j}\left(\begin{array}{c}
r \\
j
\end{array}\right)\left(\varphi A_{E}^{*}\right)^{r-j}(a \mathbb{I})^{j}+\sum_{\alpha=0}^{r}(-1)^{\alpha}\left\{\varphi^{\alpha} S_{\alpha}^{*}+J_{\alpha}^{*}(a, \varphi)\right\} \\
& \times\left\{\left(\varphi A_{E}^{*}\right)^{r-\alpha}+\sum_{k=1}^{r-\alpha}(-1)^{k}\left(\begin{array}{c}
r-\alpha \\
k
\end{array}\right)\left(\varphi A_{E}^{*}\right)^{r-\alpha-k}(a \mathbb{I})^{k}\right\} .
\end{aligned}
$$

Expanding the two brackets in 4.7 gives

$$
\begin{aligned}
T_{r}= & \varphi^{r} A_{E}^{* r}+\varphi^{r} \sum_{\alpha=1}^{r}(-1)^{\alpha} S_{\alpha}^{*} A_{E}^{* r-\alpha} \\
& +\sum_{\alpha=1}^{r}(-1)^{\alpha}\left\{J_{\alpha}^{*}(a, \varphi)\left(\varphi A_{E}^{*}-a \mathbb{I}\right)^{r-\alpha}\right. \\
& \left.+\varphi^{\alpha} S_{\alpha}^{*} \sum_{k=1}^{r-\alpha}(-1)^{k}\left(\begin{array}{c}
r-\alpha \\
k
\end{array}\right)\left(\varphi A_{E}^{*}\right)^{r-\alpha-k}(a \mathbb{I})^{k}\right\} \\
& +\sum_{j=1}^{r}(-1)^{j}\left(\begin{array}{c}
r \\
j
\end{array}\right)\left(\varphi A_{E}^{*}\right)^{r-j}(a \mathbb{I})^{j},
\end{aligned}
$$

which gives $T_{r}=\varphi^{r} T_{r}^{*}+\mathcal{N}_{r}^{*}(a, \varphi)$, and this completes the proof.

From now on, we shall write $J_{r}^{*}$ instead of $J_{r}^{*}(a, \varphi)$ and $\mathcal{N}_{r}^{*}$ instead of $\mathcal{N}_{r}^{*}(a, \varphi)$.

Next, we use Proposition 4.2 above to state the following:

Proposition 4.5. Let $M$ be a SAC half-lightlike submanifold of an almost contact metric manifold $\bar{M}$ admitting a semi-symmetric non-metric connection. Let $S_{r}^{*}$ and $T_{r}^{*}$ denote the $r$-th mean curvature and Newton transformations with respect to $A_{E}^{*}$ respectively. Then, for all $r \geq 1$ we have

$$
\begin{aligned}
\operatorname{tr}\left(T_{r}\right)= & \varphi^{r} \operatorname{tr}\left(T_{r}^{*}\right)+(-1)^{r}(n+1-r) J_{r}^{*}, \\
\operatorname{tr}\left(A_{N} \circ T_{r-1}\right)= & \varphi^{r} \operatorname{tr}\left(A_{E}^{*} \circ T_{r-1}^{*}\right)+(-1)^{r-1} r J_{r}^{*}, \\
\operatorname{tr}\left(A_{N}^{2} \circ T_{r-1}\right)= & \varphi^{r+1} \operatorname{tr}\left(A_{E}^{* 2} \circ T_{r-1}^{*}\right) \\
& +(-1)^{r}\left\{\varphi S_{1}^{*} J_{r}^{*}-\operatorname{an} \varphi^{r} S_{r}^{*}-a n J_{r}^{*}+(r+1) J_{r+1}^{*}\right\} .
\end{aligned}
$$

Proof. The proof follows by straightforward calculations.

Further, using (4.8) and 4.5 we deduce the following: 
Corollary 4.6. Let $M$ be a SAC half-lightlike submanifold of an almost contact metric manifold $\bar{M}$ admitting a semi-symmetric non-metric connection. Let $S_{r}^{*}$ and $T_{r}^{*}$ denote the $r$-th mean curvature and Newton transformations with respect to $A_{E}^{*}$ respectively. Then, for all $r \geq 1$, the trace of $\mathcal{N}_{r}^{*}$ satisfies

$$
\operatorname{tr}\left(\mathcal{N}_{r}^{*}\right)=(-1)^{r}(n+1-r) J_{r}^{*}
$$

Next, we use Theorem 4.4 to state the following:

Theorem 4.7. Let $M$ be a SAC half-lightlike submanifold of an almost contact manifold $\bar{M}$ admitting a semi-symmetric non-metric connection. Let $T_{r}$ and $T_{r}^{*}$ be the $r$-th Newton transformations corresponding to the two shape operators $A_{N}$ and $A_{E}^{*}$ respectively. Then, the operator $\mathcal{N}_{r}^{*}$ satisfies the recurrence relation

$$
\begin{aligned}
& \mathcal{N}_{1}^{*}=a n \mathbb{I}, \\
& \mathcal{N}_{r}^{*}=(-1)^{r} J_{r}^{*} \mathbb{I}-a \varphi^{r-1} T_{r-1}^{*}-a \mathcal{N}_{r-1}^{*}+\varphi A_{E}^{*} \circ \mathcal{N}_{r-1}^{*}, \quad r \geq 2 .
\end{aligned}
$$

Proof. Using the inductive formula (3.3), 4.2, 4.5), and 2.20 for a SAC halflightlike submanifold, we obtain the desired equation 4.11).

Notice that 4.11 implies the following:

Corollary 4.8. Let $M$ be a SAC half-lightlike submanifold of an almost contact manifold $\bar{M}$ admitting a semi-symmetric non-metric connection. Let $S_{r}$ and $S_{r}^{*}$ be the $r$-th mean curvatures corresponding to the two shape operators $A_{N}$ and $A_{E}^{*}$ respectively. Then, for all $r \geq 2$ we have

$$
\begin{aligned}
\operatorname{tr}\left(A_{E}^{*} \circ \mathcal{N}_{r-1}^{*}\right)= & (-1)^{r-1} \varphi^{-1}\left\{r J_{r}^{*}+a(n+2-r)\left(\varphi^{r-1} S_{r-1}^{*}+J_{r-1}^{*}\right)\right\} \\
\operatorname{tr}\left(A_{E}^{* 2} \circ \mathcal{N}_{r-1}^{*}\right)= & \varphi^{-1} \operatorname{tr}\left(A_{E}^{*} \circ \mathcal{N}_{r}^{*}\right)-a \varphi^{-1} \operatorname{tr}\left(A_{E}^{*} \circ \mathcal{N}_{r-1}^{*}\right) \\
& +a \varphi^{r-2} \operatorname{tr}\left(A_{E}^{*} \circ T_{r-1}^{*}\right)+\varphi^{-1}(-1)^{r-1} J_{r}^{*} S_{1}^{*} .
\end{aligned}
$$

Proposition 4.9. Let $(M, g, S(T M))$ be an $(n+1)$-dimensional $S A C$ half-lightlike submanifold of an indefinite nearly cosymplectic manifold $\bar{M}$ admitting a semisymmetric non-metric connection. Denote by $\nabla$ the induced connection on TM. 
Then

$$
\begin{aligned}
& g\left(\operatorname{div}^{\nabla}\left(T_{r}\right), X\right) \\
& =(-1)^{r-1} \lambda(X)\left(\varphi^{r} E\left(S_{r}^{*}\right)+E\left(\varphi^{r}\right) S_{r}^{*}+E\left(J_{r}^{*}\right)\right)+g\left(\left(\nabla_{E} A_{N}\right) T_{r-1} E, X\right) \\
& +\varphi g\left(\operatorname{div}^{\nabla}\left(\varphi^{r-1} T_{r-1}^{*}\right), A_{E}^{*} X\right)+\varphi g\left(\operatorname{div}^{\nabla}\left(\mathcal{N}_{r-1}^{*}\right), A_{E}^{*} X\right) \\
& -a g\left(\operatorname{div}^{\nabla}\left(\varphi^{r-1} T_{r-1}^{*}\right), X\right)-a g\left(\operatorname{div}^{\nabla}\left(\mathcal{N}_{r-1}^{*}\right), X\right) \\
& +a \lambda(X) \varphi^{r-1} \operatorname{tr}\left(A_{E}^{*} \circ T_{r-1}^{*}\right)+a \lambda(X) \operatorname{tr}\left(A_{E}^{*} \circ \mathcal{N}_{r-1}^{*}\right) \\
& -\varphi^{r} \lambda(X) \operatorname{tr}\left(A_{E}^{*^{2}} \circ T_{r-1}^{*}\right)-\varphi \lambda(X) \operatorname{tr}\left(A_{E}^{*^{2}} \circ \mathcal{N}_{r-1}^{*}\right) \\
& +a \tau(X) \varphi^{r-1} \operatorname{tr}\left(T_{r-1}^{*}\right)+(a-\varphi) \tau(X) \operatorname{tr}\left(\mathcal{N}_{r-1}^{*}\right) \\
& -\varphi^{r} \tau(X) \operatorname{tr}\left(A_{E}^{*} \circ T_{r-1}^{*}\right)+\left(a \eta(X)-\varphi \eta\left(A_{E}^{*} X\right)\right) \operatorname{tr}\left(\mathcal{N}_{r-1}^{*}\right) \\
& +\left(a \varphi^{r-1} \eta(X)-\varphi^{r} \eta\left(A_{E}^{*} X\right)\right) \operatorname{tr}\left(T_{r-1}^{*}\right)+\varphi^{r} \eta(X) \operatorname{tr}\left(A_{E}^{*} \circ T_{r-1}^{*}\right) \\
& +(-1)^{r-1} \eta(X) r J_{r}^{*}+\left(\varphi^{r} \lambda\left(A_{E}^{*} X\right)-a \varphi^{r-1} \lambda(X)\right) \operatorname{tr}\left(A_{E}^{*} \circ T_{r-1}^{*}\right) \\
& +\left(\varphi \lambda\left(A_{E}^{*} X\right)-a \lambda(X)\right) \operatorname{tr}\left(\mathcal{N}_{r-1}^{*}\right)+\sum_{i=0}^{n}\left\{\bar{g}\left(R\left(Z_{i}, X\right) \varphi^{r-1} T_{r-1}^{*} Z_{i}, N\right)\right. \\
& +\bar{g}\left(R\left(Z_{i}, X\right) \mathcal{N}_{r-1}^{*} Z_{i}, N\right)+\varphi^{r} \tau\left(Z_{i}\right) B\left(X, T_{r-1}^{*} Z_{i}\right) \\
& +\varphi \tau\left(Z_{i}\right) B\left(X, \mathcal{N}_{r-1}^{*} Z_{i}\right)-a \tau\left(Z_{i}\right)\left(\varphi^{r-1} g\left(X, T_{r-1}^{*} Z_{i}\right)+g\left(X, \mathcal{N}_{r-1}^{*} Z_{i}\right)\right) \\
& +\lambda\left(T_{r-1}^{*} Z_{i}\right)\left(\varphi^{r} B\left(Z_{i}, A_{E}^{*} X\right)-a \varphi^{r-1} B\left(X, Z_{i}\right)\right) \\
& +\lambda\left(\mathcal{N}_{r-1}^{*} Z_{i}\right)\left(\varphi B\left(Z_{i}, A_{E}^{*} X\right)-a B\left(X, Z_{i}\right)\right) \\
& +\left(-\varphi^{r} \lambda\left(A_{E}^{*}\left(T_{r-1}^{*} Z_{i}\right)\right)-\varphi \lambda\left(A_{E}^{*}\left(\mathcal{N}_{r-1}^{*} Z_{i}\right)\right)+a \varphi^{r-1} \lambda\left(T_{r-1}^{*} Z_{i}\right)\right. \\
& \left.+a \lambda\left(\mathcal{N}_{r-1}^{*} Z_{i}\right)\right) B\left(X, Z_{i}\right)+\left(-\varphi^{r} \eta\left(A_{E}^{*}\left(T_{r-1}^{*} Z_{i}\right)\right)-\varphi \eta\left(A_{E}^{*}\left(\mathcal{N}_{r-1}^{*} Z_{i}\right)\right)\right. \\
& \left.+a \varphi^{r-1} \eta\left(T_{r-1}^{*} Z_{i}\right)+a \lambda\left(\mathcal{N}_{r-1}^{*} Z_{i}\right)\right) g\left(X, Z_{i}\right)+\left(a \eta\left(\mathcal{N}_{r-1}^{*} Z_{i}\right)\right. \\
& \left.+a \varphi^{r-1} \eta\left(T_{r-1}^{*} Z_{i}\right)\right) g\left(X, Z_{i}\right)-\left(\varphi \eta\left(\mathcal{N}_{r-1}^{*} Z_{i}\right)\right. \\
& \left.\left.+\varphi^{r} \eta\left(T_{r-1}^{*} Z_{i}\right)\right) g\left(A_{E}^{*} X, Z_{i}\right)\right\}, \quad \forall X \in \Gamma(T M) .
\end{aligned}
$$

Proof. From (3.3), 3.14), and the fact that $A_{N}$ is self-adjoint, we derive

$$
\begin{aligned}
g\left(\operatorname{div}^{\nabla}\left(T_{r}\right), X\right)= & (-1)^{r} P X\left(S_{r}\right)+g\left(\operatorname{div}^{\nabla}\left(T_{r-1}\right), A_{N} X\right) \\
& +g\left(\sum_{\alpha=0}^{n}\left(\nabla_{Z_{\alpha}} A_{N}\right) T_{r-1} Z_{\alpha}, X\right),
\end{aligned}
$$

for all $X \in \Gamma(T M)$.

Using the definition of covariant derivative we have

$$
\begin{aligned}
g\left(\left(\nabla_{Z_{i}} A_{N}\right) T_{r-1} Z_{i}, X\right)= & g\left(T_{r-1} Z_{i},\left(\nabla_{Z_{i}} A_{N}\right) X\right)+g\left(\nabla_{Z_{i}} A_{N}\left(T_{r-1} Z_{i}\right), X\right) i \\
& -g\left(\nabla_{Z_{i}}\left(A_{N} X\right), T_{r-1} Z_{i}\right)+g\left(A_{N}\left(\nabla_{Z_{i}} X\right), T_{r-1} Z_{i}\right) \\
& -g\left(A_{N}\left(\nabla_{Z_{i}} T_{r-1} Z_{i}\right), X\right),
\end{aligned}
$$


for all $X \in \Gamma(T M)$. By virtue of $(2.11)$ and the fact that $A_{N}$ is a self-adjoint operator, equation 4.14 reduces to

$$
\begin{aligned}
g\left(\left(\nabla_{Z_{i}} A_{N}\right) T_{r-1} Z_{i}, X\right)= & g\left(T_{r-1} Z_{i},\left(\nabla_{Z_{i}} A_{N}\right) X\right) \\
& \left.+B\left(Z_{i}, A_{N} X\right) \lambda\left(T_{r-1} Z_{i}\right)+B\left(Z_{i}, T_{r-1} Z_{i}\right)\right) \lambda\left(A_{N} X\right) \\
& -B\left(Z_{i}, A_{N}\left(T_{r-1} Z_{i}\right)\right) \lambda(X)-B\left(Z_{i}, X\right) \lambda\left(A_{N}\left(T_{r-1} Z_{i}\right)\right. \\
& +\eta(X) g\left(Z_{i}, A_{N}\left(T_{r-1} Z_{i}\right)\right)-\eta\left(A_{N} X\right) g\left(Z_{i}, T_{r-1} Z_{i}\right) \\
& -\eta\left(T_{r-1} Z_{i}\right) g\left(Z_{i}, A_{N} X\right)+\eta\left(A_{N}\left(T_{r-1} Z_{i}\right)\right) g\left(Z_{i}, X\right),
\end{aligned}
$$

for any $X \in \Gamma(T M)$.

Now, applying (2.16), 2.17), 2.20), and 4.15) we derive

$$
\begin{aligned}
g\left(\left(\nabla_{Z_{i}} A_{N}\right) T_{r-1} Z_{i}, X\right)= & g\left(T_{r-1} Z_{i},\left(\nabla_{X} A_{N}\right) Z_{i}\right) \\
& +\bar{g}\left(R\left(Z_{i}, X\right) T_{r-1} Z_{i}, N\right)+\varphi\left\{\tau\left(Z_{i}\right) B\left(X, T_{r-1} Z_{i}\right)\right. \\
& \left.-\tau(X) B\left(Z_{i}, T_{r-1} Z_{i}\right)\right\}-a\left\{\tau\left(Z_{i}\right) g\left(X, T_{r-1} Z_{i}\right)\right. \\
& \left.-\tau(X) B\left(Z_{i}, T_{r-1} Z_{i}\right)\right\}+B\left(Z_{i}, A_{N} X\right) \lambda\left(T_{r-1} Z_{i}\right) \\
& \left.+B\left(Z_{i}, T_{r-1} Z_{i}\right)\right) \lambda\left(A_{N} X\right)-B\left(Z_{i}, A_{N}\left(T_{r-1} Z_{i}\right)\right) \lambda(X) \\
& -B\left(Z_{i}, X\right) \lambda\left(A_{N}\left(T_{r-1} Z_{i}\right)+\eta(X) g\left(Z_{i}, A_{N}\left(T_{r-1} Z_{i}\right)\right)\right. \\
& -\eta\left(A_{N} X\right) g\left(Z_{i}, T_{r-1} Z_{i}\right)-\eta\left(T_{r-1} Z_{i}\right) g\left(Z_{i}, A_{N} X\right) \\
& +\eta\left(A_{N}\left(T_{r-1} Z_{i}\right)\right) g\left(Z_{i}, X\right), \quad \forall X \in \Gamma(T M) .
\end{aligned}
$$

Finally, substituting 4.16 in 4.13 and using Propositions 4.2 and 4.5 and Theorem 4.4 we obtain the required equation 4.12 .

A semi-Riemannian manifold $\bar{M}$ of constant curvature $c$ is called a semi-Riemannian space form $([6,7])$ and is denoted by $\bar{M}(c)$. Then, the curvature tensor $\bar{R}$ of $\bar{M}(c)$ is given by

$$
\bar{R}(X, Y) Z=c\{\bar{g}(Y, Z) X-\bar{g}(X, Z) Y\}, \quad \forall X, Y, Z \in \Gamma(T \bar{M}) .
$$

Next, using Proposition 4.9 we have the following:

Theorem 4.10. Let $M(c)$ be $r$-totally umbilical SAC half-lightlike submanifold of constant curvature $c$ and with an integrable screen distribution $S(T M)$, of an indefinite contact manifold $\bar{M}^{n+3}$, admitting a semi-symmetric non-metric connection. Suppose that $M^{\prime}$ is a leaf of $M(c)$. If the structure vector field $\xi$ is tangent to $M(c)$, but not in $S(T M)$, then

$$
\begin{aligned}
(-1)^{r}( & \left.\varphi^{r} E\left(S_{r}^{*}\right)+E\left(\varphi^{r}\right) S_{r}^{*}+E\left(J_{r}^{*}\right)\right) \\
= & -\varphi^{r} \operatorname{tr}\left(A_{E}^{*^{2}} \circ T_{r-1}^{*}\right)-\varphi \operatorname{tr}\left(A_{E}^{* 2} \circ \mathcal{N}_{r-1}^{*}\right)-\varphi^{r} \tau(E) \operatorname{tr}\left(A_{E}^{*} \circ T_{r-1}^{*}\right) \\
& +a \operatorname{tr}\left(A_{E}^{*} \circ \mathcal{N}_{r-1}^{*}\right)+A^{\prime} \operatorname{tr}\left(T_{r-1}^{*}\right)+B^{\prime} \operatorname{tr}\left(\mathcal{N}_{r-1}^{*}\right),
\end{aligned}
$$

where $A^{\prime}=a \tau(E)-c \varphi^{r-1}$ and $B^{\prime}=(a-\varphi) \tau(E)-a-c$. 
Proof. Since $M$ is a space form, then $\operatorname{div}^{\nabla}\left(T_{r}\right) \in \Gamma\left(T M^{\perp}\right)$. Thus, taking $X=E$ in 4.12 and simplifying the resultant equation while considering 2.12 , 2.20, and (4.17), we get

$$
\begin{aligned}
(-1)^{r} & \left(\varphi^{r} E\left(S_{r}^{*}\right)+E\left(\varphi^{r}\right) S_{r}^{*}+E\left(J_{r}^{*}\right)\right)-a \operatorname{tr}\left(A_{E}^{*} \circ \mathcal{N}_{r-1}^{*}\right) \\
& +\varphi^{r} \operatorname{tr}\left(A_{E}^{* 2} \circ T_{r-1}^{*}\right)+\varphi \operatorname{tr}\left(A_{E}^{* 2} \circ \mathcal{N}_{r-1}^{*}\right)-a \varphi^{r} \tau(E) \operatorname{tr}\left(T_{r-1}^{*}\right) \\
& +(\varphi-a) \tau(E) \operatorname{tr}\left(\mathcal{N}_{r-1}^{*}\right)+\varphi^{r} \tau(E) \operatorname{tr}\left(A_{E}^{*} \circ T_{r-1}^{*}\right) \\
& +a \operatorname{tr}\left(\mathcal{N}_{r-1}^{*}\right)+c\left(\varphi^{r-1} \operatorname{tr}\left(T_{r-1}^{*}\right)+\operatorname{tr}\left(\mathcal{N}_{r-1}^{*}\right)\right)=0
\end{aligned}
$$

from which our assertion follows by re-arrangement.

From the above theorem we have:

Corollary 4.11. Let $M(c)$ be a r-totally umbilical SAC half-lightlike submanifold of constant curvature $c$ and with an integrable screen distribution $S(T M)$, of an indefinite nearly contact manifold $\bar{M}^{n+3}$, admitting a semi-symmetric non-metric connection. Suppose that $M^{\prime}$ is a leaf in $M(c)$. If the structure vector field $\xi$ is tangent to $M(c)$ and belongs to $S(T M)$, then $M(c)$ is a semi-Euclidean space if and only if

$$
\varphi^{r} E\left(S_{r}^{*}\right)+E\left(\varphi^{r}\right) S_{r}^{*}=(-1)^{r-1} \varphi^{r}\left(\operatorname{tr}\left(A_{E}^{* 2} \circ T_{r-1}^{*}\right)+\tau(E) \operatorname{tr}\left(A_{E}^{*} \circ T_{r-1}^{*}\right)\right) .
$$

Proof. The proof follows easily from Theorem 4.10 using the fact $a=0$ when $\xi \in \Gamma(S(T M))$.

From Corollary 4.11 we have

$$
\begin{aligned}
\varphi^{r} E\left(S_{r}^{*}\right)+E\left(\varphi^{r}\right) S_{r}^{*} & =\varphi^{r}\left(\operatorname{tr}\left(A_{E}^{* 2} \circ T_{r-1}^{*}\right)+\tau(E) \operatorname{tr}\left(A_{E}^{*} \circ T_{r-1}^{*}\right)\right) \\
& =r \varphi^{r} S_{r}^{*} \tau(E)+\varphi^{r} \sum_{i=1}^{n} k_{i}^{* 2} S_{r-1}^{*^{\alpha}},
\end{aligned}
$$

which on simplifying gives

$$
E\left(\varphi^{r} S_{r}^{*}\right)=r \varphi^{r} S_{r}^{*} \tau(E)+\varphi^{r} \sum_{i=1}^{n} k_{i}^{* 2} S_{r-1}^{*^{\alpha}} .
$$

Notice that 4.18) recovers Theorem 4.5 of [8], which says: For a conformal halflightlike submanifold $M(c)$ with mean curvature $K$, if $M^{\prime}$ is a totally umbilical leaf in $M(c)$, then the submanifold is a semi-Euclidean space if and only if Ksatisfies

$$
E(K)-K \tau(E)-K^{2} \varphi^{-1}=0 .
$$

\section{Special Minkowski integration formulae}

In this section, we present a new integration formula on a special SAC halflightlike submanifold $\left(M, g, S(T M), S\left(T M^{\perp}\right)\right)$ of an indefinite nearly cosymplectic manifold $(\bar{M}, \bar{g})$, called SAC $\bar{H}$-half-lightlike submanifold, via the computation of $\operatorname{div}^{\nabla}\left(T_{r} \bar{H} X^{\prime}\right)$ and $\operatorname{div}^{\nabla}\left(T_{r} \bar{H} X^{\prime}+T_{r} E\right)$, where $X^{\prime} \in \Gamma\left(S(T M)^{\perp}\right)$ and $E \in \Gamma(\operatorname{Rad} T M)$. As an application, we apply our formula in classifying minimal and r-maximal SAC half-lightlike submanifolds. We shall suppose that $M$ is 
closed and bounded (compact). An almost contact manifold $\bar{M}$ is said to be nearly cosymplectic if

$$
\left(\bar{\nabla}_{\bar{X}} \bar{\phi}\right) \bar{Y}+\left(\bar{\nabla}_{\bar{Y}} \bar{\phi}\right) \bar{X}=0
$$

for any vector fields $\bar{X}, \bar{Y}$ on $\bar{M}$, where $\bar{\nabla}$ is the connection for the semi-Riemannian metric $\bar{g}$.

Replacing $\bar{Y}$ by $\xi$ in (5.1) we obtain

$$
\bar{\nabla}_{\bar{X}} \xi=-\bar{H} \bar{X}
$$

where $\bar{H}$ is a $(1,1)$ tensor given by $\bar{H} \bar{X}=\bar{\phi}\left(\bar{\nabla}_{\xi} \bar{\phi}\right) \bar{X}$. The linear operator $\bar{H}$ has the properties $([16])$ :

$$
\begin{aligned}
\bar{H} \bar{\phi}+\bar{\phi} \bar{H} & =0, \quad \bar{H} \xi=0, \quad \eta \circ \bar{H}=0, \quad(\bar{\nabla} \times \bar{\phi}) \xi=\bar{\phi} \bar{H} X, \\
\text { and } \quad \bar{g}(\bar{H} \bar{X}, \bar{Y}) & =-\bar{g}(\bar{X}, \bar{H} \bar{Y}) \quad \text { (i.e. } \bar{H} \text { is skew-symmetric), }
\end{aligned}
$$

for all $\bar{X}, \bar{Y} \in \Gamma(T \bar{M})$.

Proposition 5.1. Let $\left(M, g, S(T M), S\left(T M^{\perp}\right)\right)$ be any half-lightlike submanifold of an indefinite nearly cosymplectic manifold $(\bar{M}, \bar{g})$. Then

$$
\bar{H} S(T M)^{\perp} \subset S(T M) .
$$

Proof. On a lightlike submanifold we have

$$
S(T M)^{\perp}=\{\operatorname{Rad} T M \oplus l \operatorname{tr}(T M)\} \perp S\left(T M^{\perp}\right) .
$$

Thus, to show that $\bar{H} S(T M)^{\perp} \subset S(T M)$ it is enough to show that

$$
\bar{H} \operatorname{Rad} T M \subset S(T M), \quad \bar{H} l \operatorname{tr}(T M) \subset S(T M), \quad \text { and } \quad \bar{H} S\left(T M^{\perp}\right) \subset S(T M) .
$$

Now, let $E \in \Gamma(\operatorname{Rad} T M)$; then by the anti-symmetry of $\bar{H}$ we have $\bar{g}(\bar{H} E, E)=0$. This shows that $\bar{H} E$ has no component along $l \operatorname{tr}(T M)$. Thus, we can see that $\bar{H} E \in \Gamma\left(T M \perp S\left(T M^{\perp}\right)\right)$, which indicates that $\bar{H} E$ has a possibility of belonging to $\Gamma(T M)$. Considering this option and the fact that on a half-lightlike submanifold $\operatorname{rank}(\operatorname{Rad} T M)=1$, we can see that $\bar{H} \operatorname{Rad} T M$ is a distribution on $M$ of $\operatorname{rank} 1$ such that $\bar{H} \operatorname{Rad} T M \cap \operatorname{Rad} T M=\{0\}$. Therefore, we can choose a particular $S(T M)$ containing $\bar{H} \operatorname{Rad} T M$ as one of its subbundles. Further, let $N \in \Gamma(l \operatorname{tr}(T M))$; then $\bar{g}(\bar{H} N, E)=-\bar{g}(N, \bar{H} E)=0$. Thus, $\bar{H} N$ has no component along $l \operatorname{tr}(T M)$. Since $\bar{g}(\bar{H} N, N)=0$ we can see that $\bar{H} N$ has no component along $\operatorname{Rad} T M$. Hence, $\bar{H} N \in \Gamma\left(S(T M) \perp S\left(T M^{\perp}\right)\right)$, from which we can also choose $\bar{H} N \in \Gamma(S(T M))$. Using a similar reasoning as above, we can see that $\bar{g}(\bar{H} W, W)=0, \bar{g}(\bar{H} W, E)=0$, and $\bar{g}(\bar{H} W, N)=0$, for $W \in \Gamma\left(S\left(T M^{\perp}\right)\right)$. From the last three equations, it is obvious that $\bar{H} W$ is neither in $\Gamma(\operatorname{Rad} T M)$ nor in $\Gamma(\operatorname{lt}(T M))$. Also, we infer that $\bar{H} W \notin \Gamma\left(S\left(T M^{\perp}\right)\right)$. In fact, if $\bar{H} W \in \Gamma\left(S\left(T M^{\perp}\right)\right)$ then there exists a nonvanishing function $\omega$ such that $\bar{H} W=\omega W$. Taking the $\bar{g}$-product of the last equation with respect to $W$ we get $0=\bar{g}(\bar{H} W, W)=\omega \bar{g}(W, W) \neq 0$, which is a contradiction. Thus, $\bar{H} W \in \Gamma(S(T M))$, which completes the proof.

Using Proposition 5.1 we have the following: 
Definition 5.2. Let $\left(M, g, S(T M), S\left(T M^{\perp}\right)\right)$ be any half-lightlike submanifold of an indefinite nearly cosymplectic manifold $(\bar{M}, \bar{g})$. We say that $M$ is $\bar{H}$-halflightlike submanifold of $\bar{M}$ if

$$
\bar{H} S(T M)^{\perp} \subset S(T M) .
$$

Unlike the non-degenerate submanifolds, in the lightlike submanifolds it is generally difficult to define certain geometric operators like divergence and Laplacian. This is attributed to the degeneracy of the induced metric tensor $g$. However, as $(M, g)$ is paracompact we can normalize it to $(M, \tilde{g})$, where

$$
\tilde{g}:=g+\lambda \otimes \lambda
$$

is a non-degenerate metric tensor. More details on the normalization process can be found in [3, 10]. With such a metric, we can successfully define the previously mentioned operators, as well as defining a special volume form $d V$ on $M$ to aid in the integration formulae that will follow. From now on, we assume that $M$ carries such a metric tensor.

Proposition 5.3. Let $\left(M, g, S(T M), S\left(T M^{\perp}\right)\right)$ be a $S A C \bar{H}$-half-lightlike submanifold of an indefinite nearly cosymplectic manifold $(\bar{M}, \bar{g})$ admitting a semisymmetric non-metric connection. Then,

$$
\begin{aligned}
& \tilde{g}\left(\nabla_{E} T_{r} \bar{H} X^{\prime}, E\right)=(-1)^{r} S_{r} \eta\left(\bar{H} X^{\prime}\right) \\
& g\left(\nabla_{Z_{i}} T_{r} \bar{H} X^{\prime}, Z_{i}\right)= g\left(\bar{H} X^{\prime},\left(\nabla_{Z_{i}} T_{r}\right) Z_{i}\right)+g\left(\nabla_{Z_{i}}^{*} T_{r} \bar{H} X^{\prime}, Z_{i}\right) \\
&-g\left(\bar{H} X^{\prime},\left(\nabla_{Z_{i}}^{*} T_{r}\right) Z_{i}\right)-\eta\left(\bar{H} X^{\prime}\right) g\left(Z_{i}, T_{r} Z_{i}\right) \\
&+(-1)^{r} S_{r} \eta\left(\bar{H} X^{\prime}\right),
\end{aligned}
$$

for all $X^{\prime} \in \Gamma\left(S(T M)^{\perp}\right)$, where $\nabla^{*}$ is the metric connection on $S(T M)$.

Proof. First, observe from 5.3 that

$$
\tilde{g}(X, E)=\bar{g}(X, N),
$$

for any $X \in \Gamma(T M)$. Thus, a straightforward calculation leads to

$$
\bar{g}\left(\nabla_{E} T_{r} \bar{H} X^{\prime}, N\right)=(-1)^{r} \bar{g}\left(\nabla_{E} S_{r} \bar{H} X^{\prime}, N\right)=(-1)^{r} \bar{g}\left(S_{r} \nabla_{E} \bar{H} X^{\prime}, N\right) .
$$

Now, applying (2.9) to the above equation we get

$$
\bar{g}\left(\nabla_{E} T_{r} \bar{H} X^{\prime}, N\right)=(-1)^{r} \bar{g}\left(S_{r} \nabla_{E}^{*} \bar{H} X^{\prime}, N\right)+(-1)^{r} S_{r} C\left(E, \bar{H} X^{\prime}\right),
$$

from which we get

$$
\bar{g}\left(\nabla_{E} T_{r} \bar{H} X^{\prime}, N\right)=(-1)^{r} S_{r} C\left(E, \bar{H} X^{\prime}\right) .
$$

Applying 2.14 to 5.7 with $X=E$ and $P Y=\bar{H} X^{\prime}$ we get

$$
\bar{g}\left(\nabla_{E} T_{r} \bar{H} X^{\prime}, N\right)=(-1)^{r} S_{r} \eta\left(\bar{H} X^{\prime}\right),
$$

which, if considered with (5.6), proves (5.4). On the other hand, using 2.11) we derive

$$
g\left(\nabla_{Z_{i}} T_{r} \bar{H} X^{\prime}, Z_{i}\right)=Z_{i}\left(g\left(T_{r} \bar{H} X^{\prime}, Z_{i}\right)\right)-g\left(\bar{H} X^{\prime}, T_{r} \nabla_{Z_{i}} Z_{i}\right) .
$$


Now, applying the definition of covariant derivative of $T_{r}$ on 5.8 we get

$$
\begin{aligned}
g\left(\nabla_{Z_{i}} T_{r} \bar{H} X^{\prime}, Z_{i}\right)= & g\left(\bar{H} X^{\prime},\left(\nabla_{Z_{i}} T_{r}\right) Z_{i}\right)+Z_{i}\left(g\left(T_{r} \bar{H} X^{\prime}, Z_{i}\right)\right) \\
& -g\left(\bar{H} X^{\prime}, \nabla_{Z_{i}} T_{r} Z_{i}\right)+(-1)^{r} S_{r} \eta\left(\bar{H} X^{\prime}\right) \\
& +\eta\left(Z_{i}\right) g\left(\bar{H} X^{\prime}, T_{r} Z_{i}\right) .
\end{aligned}
$$

Also, using 2.11 we derive

$$
\begin{aligned}
& Z_{i}\left(g\left(\bar{H} X^{\prime}, T_{r} Z_{i}\right)\right)-g\left(\bar{H} X^{\prime}, \nabla_{Z_{i}} T_{r} Z_{i}\right) \\
& \quad=g\left(T_{r} \nabla_{Z_{i}} \bar{H} X^{\prime}, Z_{i}\right)-\eta\left(\bar{H} X^{\prime}\right) g\left(Z_{i}, T_{r} Z_{i}\right)-\eta\left(Z_{i}\right) g\left(Z_{i}, T_{r} \bar{H} X^{\prime}\right) .
\end{aligned}
$$

Then substituting $(5.10)$ in $(5.9)$ and then applying $(2.9)$ we get $(5.5)$, which completes the proof.

Now we are ready to compute $\operatorname{div}^{\nabla}\left(T_{r} \bar{H} X^{\prime}\right)$.

Theorem 5.4. Let $\left(M, g, S(T M), S\left(T M^{\perp}\right)\right)$ be a $S A C \bar{H}$-half-lightlike submanifold of an indefinite nearly cosymplectic manifold $(\bar{M}, \bar{g})$ admitting a semi-symmetric non-metric connection. Then,

$$
\begin{aligned}
\operatorname{div}^{\nabla}\left(T_{r} \bar{H} X^{\prime}\right)= & g\left(\operatorname{div}^{\nabla}\left(\varphi^{r} T_{r}^{*}\right), \bar{H} X^{\prime}\right)+g\left(\operatorname{div}^{\nabla}\left(\mathcal{N}_{r}^{*}\right), \bar{H} X^{\prime}\right) \\
& -g\left(\operatorname{div}^{\nabla^{*}}\left(\varphi^{r} T_{r}^{*}\right), \bar{H} X^{\prime}\right)-g\left(\operatorname{div}^{\nabla^{*}}\left(\mathcal{N}_{r}^{*}\right), \bar{H} X^{\prime}\right) \\
& +\operatorname{tr}\left(\nabla^{*}\left(\varphi^{r} T_{r}^{*} \bar{H} X^{\prime}\right)\right)+\operatorname{tr}\left(\nabla^{*}\left(\mathcal{N}_{r}^{*} \bar{H} X^{\prime}\right)\right) \\
& -\varphi^{r} \eta\left(\bar{H} X^{\prime}\right) \operatorname{tr}\left(T_{r}^{*}\right)-\eta\left(\bar{H} X^{\prime}\right) \operatorname{tr}\left(\mathcal{N}_{r}^{*}\right) \\
& +(-1)^{r}(n+1) \eta\left(\bar{H} X^{\prime}\right)\left(\varphi^{r} S_{r}^{*}+J_{r}^{*}\right),
\end{aligned}
$$

for all $X^{\prime} \in \Gamma\left(S(T M)^{\perp}\right)$.

Proof. By definition of divergence we have

$$
\begin{aligned}
\operatorname{div}^{\nabla}\left(T_{r} \bar{H} X^{\prime}\right) & =\operatorname{tr}\left(\nabla T_{r} \bar{H} X^{\prime}\right) \\
& =\sum_{i=1}^{n} g\left(\nabla_{Z_{i}} T_{r} \bar{H} X^{\prime}, Z_{i}\right)+\tilde{g}\left(\nabla_{E} T_{r} \bar{H} X^{\prime}, E\right) .
\end{aligned}
$$

Now, applying (5.4) and 5.5 of Proposition 5.3 to 5.11 we get the desired equation, which ends the proof.

Theorem 5.5. Let $\left(M, g, S(T M), S\left(T M^{\perp}\right)\right)$ be a $S A C \bar{H}$-half-lightlike submanifold of an indefinite nearly cosymplectic manifold $(\bar{M}, \bar{g})$ admitting a semi-symmetric non-metric connection. Then

$$
\operatorname{div}^{\nabla}\left(T_{r} E\right)=(-1)^{r}\left(A_{1} S_{r}^{*}+A_{2} J_{r}^{*}+\varphi^{r} E\left(S_{r}^{*}\right)+E\left(J_{r}^{*}\right)\right),
$$

where

$$
A_{1}=\varphi^{r} \tau(E)+E\left(\varphi^{r}\right)-\varphi^{r} S_{1}^{*} \quad \text { and } \quad A_{2}=\tau(E)-S_{1}^{*},
$$

for any $E \in \Gamma(\operatorname{Rad} T M)$. 
Proof. By straightforward calculations we have

$$
\operatorname{div}^{\nabla}\left(T_{r} E\right)=\operatorname{tr}\left(\nabla T_{r} E\right)=\sum_{i=1}^{n} g\left(\nabla_{Z_{i}} T_{r} E, Z_{i}\right)+\tilde{g}\left(\nabla_{E} T_{r} E, E\right) .
$$

But

$$
\tilde{g}\left(\nabla_{E} T_{r} E, E\right)=\bar{g}\left(\nabla_{E} T_{r} E, N\right)=(-1)^{r}\left(E\left(S_{r}\right)+S_{r} \bar{g}\left(\nabla_{E} E, N\right)\right) .
$$

Now, applying (2.10) and Proposition 4.2 to (5.13) we get

$$
\bar{g}\left(\nabla_{E} T_{r} E, N\right)=(-1)^{r}\left(E\left(\varphi^{r} S_{r}^{*}\right)+E\left(J_{r}^{*}\right)+\tau(E)\left(\varphi^{r} S_{r}^{*}+J_{r}^{*}\right)\right) .
$$

Also,

$$
g\left(\nabla_{Z_{i}} T_{r} E, Z_{i}\right)=(-1)^{r-1}\left(\varphi^{r} S_{r}^{*}+J_{r}^{*}\right) g\left(A_{E}^{*} Z_{i}, Z_{i}\right) .
$$

Finally, replacing (5.13) and (5.14) in (5.12), we get the desired result.

From Theorems 5.4 and 5.5 we have:

Theorem 5.6. Let $\left(M, g, S(T M), S\left(T M^{\perp}\right)\right)$ be a $S A C \bar{H}$-half-lightlike submanifold of an indefinite nearly cosymplectic manifold $(\bar{M}, \bar{g})$ admitting a semi-symmetric non-metric connection. Then,

$$
\begin{aligned}
\operatorname{div}^{\nabla}\left(T_{r}\left(\bar{H} X^{\prime}+E\right)\right)= & g\left(\operatorname{div}^{\nabla}\left(\varphi^{r} T_{r}^{*}\right), \bar{H} X^{\prime}\right)+g\left(\operatorname{div}^{\nabla}\left(\mathcal{N}_{r}^{*}\right), \bar{H} X^{\prime}\right) \\
& -g\left(\operatorname{div}^{\nabla^{*}}\left(\varphi^{r} T_{r}^{*}\right), \bar{H} X^{\prime}\right)-g\left(\operatorname{div}^{\nabla^{*}}\left(\mathcal{N}_{r}^{*}\right), \bar{H} X^{\prime}\right) \\
& +\operatorname{tr}\left(\nabla^{*}\left(\varphi^{r} T_{r}^{*} \bar{H} X^{\prime}\right)\right)+\operatorname{tr}\left(\nabla^{*}\left(\mathcal{N}_{r}^{*} \bar{H} X^{\prime}\right)\right) \\
& +(-1)^{r}\left(B_{1} S_{r}^{*}+B_{2} J_{r}^{*}+\varphi^{r} E\left(S_{r}^{*}\right)+E\left(J_{r}^{*}\right)\right),
\end{aligned}
$$

where

$$
B_{1}=A_{1}+\varphi^{r} r \eta\left(\bar{H} X^{\prime}\right) \text { and } B_{2}=A_{2}+r \eta\left(\bar{H} X^{\prime}\right),
$$

for all $X^{\prime} \in \Gamma\left(S(T M)^{\perp}\right)$.

Proof. The proof follows directly from Theorems 5.4 and 5.5 equation 4.8 and the fact that $\operatorname{div}^{\nabla}\left(T_{r}\left(\bar{H} X^{\prime}+E\right)\right)=\operatorname{div}^{\nabla}\left(T_{r} \bar{H} X^{\prime}\right)+\operatorname{div}^{\nabla}\left(T_{r} E\right)$.

From the above theorem we have the following:

Theorem 5.7. Let $\left(M, g, S(T M), S\left(T M^{\perp}\right)\right)$ be a compact $S A C \bar{H}$-half-lightlike submanifold of an indefinite nearly cosymplectic manifold $(\bar{M}, \bar{g})$ admitting a semisymmetric non-metric connection. Let $\mathrm{d} V$ denote a volume form on $M$ with respect to $\tilde{g}$. Then,

$$
\begin{aligned}
(-1)^{r-1} & \int_{M}\left(B_{1} S_{r}^{*}+B_{2} J_{r}^{*}+\varphi^{r} E\left(S_{r}^{*}\right)+E\left(J_{r}^{*}\right)\right) \mathrm{d} V \\
= & \int_{M} g\left(\operatorname{div}^{\nabla}\left(\varphi^{r} T_{r}^{*}\right), \bar{H} X^{\prime}\right) \mathrm{d} V+\int_{M} g\left(\operatorname{div}^{\nabla}\left(\mathcal{N}_{r}^{*}\right), \bar{H} X^{\prime}\right) \mathrm{d} V \\
& \quad-\int_{M} g\left(\operatorname{div}^{\nabla^{*}}\left(\varphi^{r} T_{r}^{*}\right), \bar{H} X^{\prime}\right) \mathrm{d} V-\int_{M} g\left(\operatorname{div}^{\nabla^{*}}\left(\mathcal{N}_{r}^{*}\right), \bar{H} X^{\prime}\right) \mathrm{d} V \\
& +\int_{M} \operatorname{tr}\left(\nabla^{*}\left(\varphi^{r} T_{r}^{*} \bar{H} X^{\prime}\right)\right) \mathrm{d} V+\int_{M} \operatorname{tr}\left(\nabla^{*}\left(\mathcal{N}_{r}^{*} \bar{H} X^{\prime}\right)\right) \mathrm{d} V
\end{aligned}
$$


where

$$
B_{1}=A_{1}+\varphi^{r} r \eta\left(\bar{H} X^{\prime}\right) \quad \text { and } \quad B_{2}=A_{2}+r \eta\left(\bar{H} X^{\prime}\right),
$$

for all $X^{\prime} \in \Gamma\left(S(T M)^{\perp}\right)$.

Proof. Since $M$ is compact, then applying Stokes' theorem we see that

$$
\int_{M} \operatorname{div}^{\nabla}\left(T_{r}\left(\bar{H} X^{\prime}+E\right)\right) \mathrm{d} V=0, \quad \forall X^{\prime} \in \Gamma\left(S(T M)^{\perp}\right) .
$$

Now, using (5.15) and Theorem 5.6 we get the desired result.

In particular, if $\bar{M}$ is an indefinite cosymplectic manifold (i.e., $\bar{H}=0$ ), then

$$
\int_{M}\left(B_{1} S_{r}^{*}+B_{2} J_{r}^{*}+\varphi^{r} E\left(S_{r}^{*}\right)+E\left(J_{r}^{*}\right)\right) \mathrm{d} V=0 .
$$

Corollary 5.8. Let $\left(M, g, S(T M), S\left(T M^{\perp}\right)\right)$ be a compact $S A C \bar{H}$-half-lightlike submanifold of an indefinite nearly cosymplectic manifold $(\bar{M}, \bar{g})$ admitting a semisymmetric non-metric connection. If $\xi \in \Gamma(S(T M))$, then

$$
\begin{aligned}
(-1)^{r-1} \int_{M}( & \left.B_{1} S_{r}^{*}+\varphi^{r} E\left(S_{r}^{*}\right)\right) \mathrm{d} V=\int_{M} g\left(\operatorname{div}^{\nabla}\left(\varphi^{r} T_{r}^{*}\right), \bar{H} X^{\prime}\right) \mathrm{d} V \\
& -\int_{M} g\left(\operatorname{div}^{\nabla^{*}}\left(\varphi^{r} T_{r}^{*}\right), \bar{H} X^{\prime}\right) \mathrm{d} V+\int_{M} \operatorname{tr}\left(\nabla^{*}\left(\varphi^{r} T_{r}^{*} \bar{H} X^{\prime}\right)\right) \mathrm{d} V,
\end{aligned}
$$

for all $X^{\prime} \in \Gamma\left(S(T M)^{\perp}\right)$.

When $M$ is a SAC $\bar{H}$-half-lightlike submanifold of constant sectional curvature, then $\operatorname{div}^{\nabla}\left(T_{r}\right) \in \Gamma\left(T M^{\perp}\right)$ and hence we have the following:

Corollary 5.9. Let $\left(M, g, S(T M), S\left(T M^{\perp}\right)\right)$ be a compact $S A C \bar{H}$-half-lightlike submanifold of constant curvature of an indefinite nearly cosymplectic manifold $(\bar{M}, \bar{g})$ admitting a semi-symmetric non-metric connection. Then,

$$
\begin{aligned}
(-1)^{r-1} & \int_{M}\left(B_{1} S_{r}^{*}+B_{2} J_{r}^{*}+\varphi^{r} E\left(S_{r}^{*}\right)+E\left(J_{r}^{*}\right)\right) \mathrm{d} V \\
= & -\int_{M} g\left(\operatorname{div}^{\nabla^{*}}\left(\varphi^{r} T_{r}^{*}\right), \bar{H} X^{\prime}\right) \mathrm{d} V-\int_{M} g\left(\operatorname{div}^{\nabla^{*}}\left(\mathcal{N}_{r}^{*}\right), \bar{H} X^{\prime}\right) \mathrm{d} V \\
& +\int_{M} \operatorname{tr}\left(\nabla^{*}\left(\varphi^{r} T_{r}^{*} \bar{H} X^{\prime}\right)\right) \mathrm{d} V+\int_{M} \operatorname{tr}\left(\nabla^{*}\left(\mathcal{N}_{r}^{*} \bar{H} X^{\prime}\right)\right) \mathrm{d} V
\end{aligned}
$$

for all $X^{\prime} \in \Gamma\left(S(T M)^{\perp}\right)$.

Some applications of Theorem 5.7. Integral formulae are very important in differential geometry since they provide obstructions to the existence of foliations whose leaves enjoy some special geometric properties, such as being totally geodesic (or totally umbilic), minimal, constant mean curvature, and many more. Most of the interesting and useful integral formulae in both Riemannian and semiRiemannian geometry are obtained by computing the divergence of certain vector fields and applying Stokes' theorem (see some examples in [2]). Such integral formulae have also been studied in degenerate submanifolds, particularly on null hypersurfaces (see [3, 5]). 
A half-lightlike submanifold $(M, g)$ is said to be minimal if $\mathcal{S}_{1}^{*}=0$. Moreover, if $\mathcal{S}_{r}^{*}=0$, for all $r \geq 1$, then we say that $M$ is $r$-maximal [3]. Let us suppose that $\tau(E)=0$, that is, the local section $E$ is a geodesic [7], and that $\xi$ is tangent to $M$. Furthermore, we assume that $\bar{M}$ is a normal nearly cosymplectic manifold, in which $\bar{H}=0$. Under these assumptions, $B_{1}=A_{1}=E\left(\varphi^{r}\right)-\varphi^{r} \mathcal{S}_{1}^{*}, B_{2}=A_{2}=-\mathcal{S}_{1}^{*}$, and $J_{r}^{*}=0$. Thus, the integral formula of Theorem 5.7 reads as

$$
\int_{M}\left(\left(E\left(\varphi^{r}\right)-\varphi^{r} \mathcal{S}_{1}^{*}\right) S_{r}^{*}+\varphi^{r} E\left(\mathcal{S}_{r}^{*}\right)\right) \mathrm{d} V=0
$$

for all $r \geq 1$. Setting $r=1$ in the above formula and then using Corollary 4.11 , we get

$$
\int_{M} \varphi \mathcal{S}_{1}^{* 2} \mathrm{~d} V=0
$$

In the case that $\varphi$ is a constant function, we get $\mathcal{S}_{1}^{*}=0$, that is $M$ is minimal. In addition to this, if $\operatorname{tr}\left(A_{E}^{* 2} \circ T_{r-1}^{*}\right)$ is a non-negative function on $M$, then (3.13), (5.16) and Corollary 4.11 imply that $\mathcal{S}_{r}^{*}=0$. That is, $M$ is $r$-minimal. Thus, based on the above discussion we have the following characterization result.

Theorem 5.10. Let $(M, g)$ be a totally umbilical $S A C \bar{H}$-half-lightlike submanifold of a normal nearly cosymplectic manifold $(\bar{M}, \bar{g})$, such that $E$ is a geodesic and that $\xi$ is tangent to $M$. Then, $M$ is a minimal submanifold of $\bar{M}$. Moreover, if $\operatorname{tr}\left(A_{E}^{* 2} \circ T_{r-1}^{*}\right)$ is a non-negative function on $M$, then $M$ is $r$-maximal.

\section{REFERENCES}

[1] K. Andrzejewski, W. Kozlowski and K. Niedziałomski, Generalized Newton transformation and its applications to extrinsic geometry, Asian J. Math. 20 (2016), no. 2, 293-322. MR 3480021

[2] K. Andrzejewski and P. G. Walczak, The Newton transformation and new integral formulae for foliated manifolds, Ann. Global Anal. Geom. 37 (2010), no. 2, 103-111. MR 2578259.

[3] C. Atindogbé and H. T. Fotsing, Newton transformations on null hypersurfaces, Commun. Math. 23 (2015), no. 1, 57-83. MR 3394078

[4] D.E. Blair, Riemannian Geometry of Contact and Symplectic Manifolds, Progress in Mathematics, 203. Birkhäuser Boston, Boston, MA, 2002. MR 1874240

[5] J. Dong and X. Liu, Totally umbilical lightlike hypersurfaces in Robertson-Walker spacetimes, ISRN Geom. 2014, Art. ID 974695, 10 pp. MR 3187030.

[6] K. L. Duggal and A. Bejancu, Lightlike Submanifolds of Semi-Riemannian Manifolds and Applications, Mathematics and Its Applications, 364. Kluwer Academic Publishers, Dordrecht, 1996. MR 1383318

[7] K. L. Duggal and B. Sahin, Differential Geometry of Lightlike Submanifolds. Frontiers in Mathematics, Birkhäuser Verlag, Basel, 2010. MR 2598375

[8] K. L. Duggal and B. Sahin, Screen conformal half-lightlike submanifolds, Int. J. Math. Math. Sci. 2004, no. 68, 3737-3753. MR 2128788

[9] R. S. Gupta and A. Sharfuddin, Generalised Cauchy-Riemann lightlike submanifolds of indefinite Kenmotsu manifolds, Note Mat. 30 (2010) no. 2, 49-59. MR 2943023

[10] M. Gutiérrez and B. Olea, Induced Riemannian structures on null hypersurfaces, Math. Nachr. 289 (2016), no. 10, 1219-1236. MR 3520713

[11] D. H. Jin, Non-existence of lightlike submanifolds of indefinite trans-Sasakian manifolds with non-metric $\theta$-connections, Commun. Korean Math. Soc. 30 (2015), no. 1, 35-43. MR 3313449.

[12] F. Massamba, Totally contact umbilical lightlike hypersurfaces of indefinite Sasakian manifolds, Kodai Math. J. 31 (2008), 338-358. MR 2475274 
[13] F. Massamba, On semi-parallel lightlike hypersurfaces of indefinite Kenmotsu manifolds, J. Geom. 95 (2009), no. 1-2, 73-89. MR 2595664

[14] F. Massamba, On lightlike geometry in indefinite Kenmotsu manifolds. Math. Slovaca 62 (2012), no. 2, 315-344. MR 2891725

[15] F. Massamba, Screen almost conformal lightlike geometry in indefinite Kenmotsu space forms, Int. Electron. J. Geom. 5 (2012), no. 2, 36-58. MR 3161052

[16] F. Massamba and S. Ssekajja, Some remarks on quasi-generalized CR-null geometry in indefinite nearly cosymplectic manifolds, Int. J. Math. Math. Sci. 2016, Art. ID 9613182, 10 pp. MR 3576087.

[17] E. Yaşar, A. C. Çöken, and A. Yücesan, Lightlike hypersurfaces in semi-Riemannian manifold with semi-symmetric non-metric connection, Math. Scand. 102 (2008), no. 2, 253-264. MR 2437189

F. Massamba ${ }^{凶}$

School of Mathematics, Statistics and Computer Science, University of KwaZulu-Natal, Private Bag X01, Scottsville 3209, South Africa

massfort@yahoo.fr, Massamba@ukzn.ac.za

S. Ssekajja

School of Mathematics, Statistics and Computer Science, University of KwaZulu-Natal, Private Bag X01, Scottsville 3209, South Africa

ssekajja.samuel.buwaga@aims-senegal.org

Received: October 31, 2017

Accepted: September 11, 2018 